\title{
The autonomic nervous system and cardiac arrhythmias: current concepts and emerging therapies
}

Neil Herring ${ }^{1,2 *}$, Manish Kalla² and David J. Paterson ${ }^{2 *}$

${ }^{1}$ Oxford University Hospital NHS Foundation Trust, Oxford Heart Centre, John Radcliffe Hospital, Oxford, UK. ${ }^{2}$ Burdon Sanderson Cardiac Science Centre, Department of Physiology, Anatomy and Genetics, University of Oxford, UK.

*e-mail: neilherring@doctors.org.uk and david.paterson@dpag.ox.ac.uk

Abstract | Research into cardiac autonomic control has received an explosion of interest in the past 20 years, and we are now at a critical juncture with regard to the clinical translation of the experimental findings. There has been a rush to develop clinical interventions and implant a range of devices aimed at cardiac neuromodulation therapy. This interest has been driven by research, superimposed on commercial opportunities and perhaps the more relaxed regulatory framework governing implantable devices and interventions compared with that for pharmacotherapy. However, many of the results of the clinical trials into these therapies have been disappointing or conflicting. This lack of positive results is partly due to a scramble to find simple solutions for complex problems that we do not yet fully understand. Are there reasons to be optimistic? In this Review, we highlight areas in the field of cardiac autonomic control that we feel show most promise for clinical translation, and areas where our current range of blunt tools need to be refined to bring about long-term success in treating arrhythmia. 


\section{[H1] Introduction}

Neurologists have known for many years that brain injury can cause cardiovascular complications ${ }^{1}$. These complications can range from disturbances in blood pressure to marked alterations in the electrocardiogram leading to cardiac arrhythmias. But even without neurological trauma, the autonomic nervous system (ANS) is a powerful modulator of cardiac excitability when the body faces physiological, psychological and pathophysiological stress ${ }^{2}$, given the neural connection between the brain and the heart, and the heart's own intrinsic nervous system - the 'little brain in the heart' ${ }^{3}$. Indeed, the origins of emotions controlling the heart and the heart sensing emotion are embedded in folklore and literature; 'frightened to death', 'died of a broken heart', 'matters of the heart', heart-felt'. Is this 'neuro-mythology' or is there a neurophysiological basis to these sayings that presents a therapeutic opportunity to target the cardiacneural axis?

During the past 45 years, our improved understanding of the neuroanatomical circuits at the level of the heart has resulted in the re-emergence of the idea that guided therapy to the ANS can be effective in treating arrhythmia. These therapeutic strategies have involved either neurostimulation, surgical resection or ablation of ganglia, or gene therapy that is targeted to cardiac neuro-effector sites ${ }^{4,5,6,7,8,9}$ (Figure 1 ). This Review discusses the neuroanatomical and neurophysiological basis for neuromodulation therapy, and also assesses the latest clinical trial data on the clinical efficacy of this approach.

\section{[H1] Integrated cardiac neuroanatomy}

The ANS is responsible for fine tuning the activity of the heart to meet the needs of the entire circulatory system and organism, as its behaviour varies during periods of rest, stress and exercise ${ }^{10}$. The classical view of the sympathetic and parasympathetic branches of the ANS functioning as the 'accelerator and brake' are over simplistic given what we now know about the complex neuroanatomy and physiology of the ANS ${ }^{11}$. A series of elegant experiments, pioneered by investigators such as Ardell and Armour ${ }^{3}$, have revealed an interconnected cardio-neural hierarchy. As shown in Figure 2, level 1 in this hierarchy includes higher cortical centres as well as the brainstem and the spinal cord. Level 2 includes all intrathoracic extracardiac neurons (including the stellate ganglia [G] ${ }^{12,13}$, and level 3 encompasses all the intrinsic cardiac neurons ${ }^{3}$. Afferents within the heart and circulation provide sensory signals to levels 1 and 2 for processing ${ }^{14,15}$. This way, the excitability of efferent cardiac parasympathetic or sympathetic pathways therefore depends on tonic inputs at several levels in the brain, spinal cord and in the extrinsic and intrinsic cardiac ganglia ${ }^{16}$, as illustrated in Figure 2. 
In the setting of some forms of hypertension, acute myocardial infarction (MI) and chronic heart failure (HF), neurophysiological changes occur at all levels of the neural hierarchy ${ }^{17,18}$. At the level of the heart, scar formation and fibrosis ${ }^{19}$, particularly in the border zone of infarcts ${ }^{20}$, contribute to conduction block ${ }^{21}$ and slowly conducting pathways lengthened by branching and merging bundles of surviving cardiomyocytes ${ }^{22}$. These alterations create a fixed substrate for re-entrant arrhythmia. In addition, neural remodelling and heterogeneity of innervation can also superimpose a dynamic arrhythmogenic substrate ${ }^{23-25}$. Afferentmediated activation of neurohumoral systems also increases sympathetic drive and reduces vagal tone ${ }^{26}$, which in the short term can facilitate the maintenance of cardiac output ${ }^{27}$. However, this maintenance of cardiac output is at the expense of increased myocardial oxygen demand and $\mathrm{Ca}^{2+}$ overload in cardiomyocytes, leading to ectopic activity and structural remodelling, causing hypertrophy. Chronic, abnormal cardiac afferent signalling reinforces the maladaptive response of persistent sympathetic activity ${ }^{28}$ and loss of cardiovagal protection ${ }^{29}$, leading to the progression of cardiac disease, including increased likelihood of pump failure and arrhythmia-induced sudden death.

Although these sweeping statements are broadly true, the complexity within the 'heart's little brain', and indeed at higher levels of the neural hierarchy, should not be underestimated. The neuronal connections are not as simple as highlighted in diagrams such as the one shown in Figure 2, although these signalling loops undoubtedly exist. The neural circuits underpinning these pathways are still poorly represented in 3D reconstructions and the electrophysiological characterization in normal and diseased states is incomplete (Figures 3 and 4). Immunohistochemistry shows that neurons within the intrinsic cardiac plexus are mostly cholinergic, phenotypically complex, and stimulation at discrete sites identifies clusters capable of independent and selective effects on cardiac electrophysiology ${ }^{30}$. Neuronal recordings and immunostaining demonstrate the presence within the intrinsic cardiac plexus of local circuit neurons and afferents that respond to different mechanical and chemical stimuli, as well as parasympathetic ${ }^{31,32}$ and a few sympathetic ${ }^{33}$ efferents.

The principal cells in the intracardiac ganglia send axonal projections to pacemaker cells, the cardiac electrical conduction system and the contracting myocardium. Within the ganglia, some cells are interneurons that terminate in the ganglion itself, while other axons innervate other ganglia within the plexus. Sympathetic postganglionic efferents pass through and can also terminate within the intracardiac ganglia. Moreover, the ganglia receive a rich afferent innervation, and together with small intensively fluorescent cells that interact with interneurons and principal cells, collectively add to the network complexity ${ }^{34}$. Furthermore, the network can remodel both structurally $y^{35}$ and behaviourally ${ }^{36}$ in disease states (Figure 5a). For example, epicardial application of resiniferatoxin to activate and destroy cardiac afferents that express vanilloid receptor 1 (VR1) enhances sympathetic outflow in normal pigs ${ }^{37}$. Yet in a rodent model of chronic $\mathrm{HF}$, the same intervention reduces cardiac and renal sympathetic nerve activity and improves 
cardiac remodelling ${ }^{29,38}$. Moreover, the physiology of different neurotransmitters and co-transmitters cross communicating between different neuronal populations further complicates data interpretation. Following myocardial injury, the process of reinnervation and the release kinetics of classical neurotransmitters and co-transmitters, superimposed on neurotransmitter switching, are only just beginning to be appreciated $^{16,39,40}$.

\section{[H1] The ANS and ventricular arrhythmias}

Given the millions of normal heart beats that occur over a lifetime, it seems surprising that even in patients with cardiac pathology only on very rare occasions are abnormal rhythms generated. Even then, these abnormal rhythms can be brief and asymptomatic whereas on other occasions, they can be sustained and lifethreatening. A useful framework for understanding arrhythmias is that they initially require a trigger to generate an extra-stimulus. This extra-stimulus needs to have the right properties and be perfectly timed within a vulnerable window to generate an ectopic beat. Then, a suitable substrate needs to be present within the heart to maintain the propagation of the ectopic beat. The substrate can be structural and/or electrophysiological and static and/or dynamic. Ventricular tachycardia (VT) is formed from a single re-entrant circuit, but if fragmentation or wave-break occurs, then the single spiral can form multiple wavelets and fibrillation $^{41,42}$.

Each step in the process of arrhythmogenesis is probabilistic, with all criteria only rarely being fulfilled, although the ANS can influence every stage ${ }^{43}$. Overall, both acute MI and chronic HF are associated with sympathetic hyperactivity and vagal withdrawal in experimental animal models $s^{44-47}$ and large-scale human studies. In human studies, plasma catecholamine levels, heart rate variability and baroreflex sensitivity are predictive of morbidity and mortality $4^{48,49,50}$. In these conditions, ventricular arrhythmias tend to occur during exercise, first thing in the morning or during REM sleep when sympathetic drive is highest ${ }^{51,52}$. Baroreflex sensitivity ${ }^{53}$ and heart rate recovery following exercise ${ }^{54}$ are also important modifiers of the risk of arrhythmia in patients with long QT syndrome (LQTS) type 1 (which is due to mutations in KNCQ1, encoding the channel responsible for the slow component of the delayed rectifier $\mathrm{K}^{+}$current, $l_{\mathrm{Ks}}$ ). Thus, understanding spatiotemporal features of changes in cardiac innervation continue to be the subject of study as investigators probe neural targets as an alternative therapeutic approach in modifying the risk of ventricular arrhythmia.

[H2] Arrhythmic triggers and B-adrenergic receptor stimulation. Arrhythmic triggers include afterdepolarisation and abnormal automaticity. The latter can be driven by $\mathrm{Ca}^{2+}$ release from the sarcoplasmic reticulum, which drives membrane depolarization, or by local currents from ischaemic regions that depolarize 
neighbouring zones. Most pathological arrhythmias are triggered by a delayed afterdepolarization (DAD), which results from an overloaded $\mathrm{Ca}^{2+}$ store in the sarcoplasmic reticulum that causes spontaneous diastolic $\mathrm{Ca}^{2+}$ release driving electrogenic $\mathrm{Na}-\mathrm{Ca}^{2+}$ exchange (NCX) current, which then depolarizes the membrane towards the action potential threshold ${ }^{55,56}$. This $\mathrm{Ca} 2+$ overload is exacerbated by $\beta$-adrenergic receptor stimulation of adenylyl cyclase ${ }^{57}$ leading to elevations in cAMP levels ${ }^{58}$, which in turn increases intracellular $\mathrm{Ca}^{2+}$ loading via protein kinase A (PKA)-mediated increase in the L-type $\mathrm{Ca}^{2+}$ current $\left(I_{\mathrm{CaL}}\right)^{59}$ and removal of phospholamban-mediated inhibition of the sarcoplasmic reticulum $\mathrm{Ca}^{2+}$ ATPase 2 (SERCA2) ${ }^{60}$. In the border zone of a MI scar, remodelling of ion channels, transporters and $\mathrm{Ca}^{2+}$ calmodulin dependent kinase II (CaMKII) signalling influences $\mathrm{Ca}^{2+}$ handling ${ }^{61}$ and can predispose to an arrhythmic trigger ${ }^{62}$.

Early afterdepolarizations (EADs) are more common when the action potential is prolonged, as occurs in inherited LQTS, hypokalaemia and hypocalcaemia, or in the presence of drugs that block $\mathrm{K}^{+}$channels. EADs are more common during extremes of bradycardia, when the action potential is longer or during episodes of tachycardia if the action potential duration (APD) is not shortened, and are, therefore, influenced by autonomic control of heart rate and $A \mathrm{PD}^{63}$. The mechanism for EADs during the plateau phase of the ventricular action potential is classically thought to be due to reactivation of the $I_{\text {cal }}{ }^{55,64}$. Like DADs, EADs are also more common during conditions of intracellular $\mathrm{Ca}^{2+}$ overload, because the $I_{\mathrm{Cat}}$ can be enhanced by high intracellular $\mathrm{Ca}^{2+}$ levels and by L-type $\mathrm{Ca}^{2+}$ channel phosphorylation by CaMKII ${ }^{65}$ and can, therefore, also be influenced by the ANS independently of the heart rate.

[H2] Re-entry and adrenergic modulation of refractoriness and restitution. Atrial or ventricular ectopic beats are common in otherwise normal healthy hearts, in which the ectopic beats rarely lead to arrhythmias. The ectopic beats have to be perfectly timed during a vulnerable window and need to have a suitable substrate such that the wave of excitation they produce can form a self-sustaining circuit (circus or spiral wave). Cardiomyocytes emerging from their refractory period [G] are then re-excited prematurely by the return of the local excitation wave (re-entry). A slow conduction velocity of wave propagation favours re-entry, as does a short refectory period, because it allows time for the cardiomyocytes to regain excitability. $\beta$ Adrenergic receptor stimulation is well known to shorten the refractory period by increasing the $I_{\mathrm{ks}}$ and shortening the $A P D^{66}$, and the effects of stellectomy and vagotomy on ventricular refractoriness were first described in vivo in dog models ${ }^{67}$.

The shorter the APD and refractory period of an ectopic beat, the more likely it is to be able to produce a reentrant circuit. The relationship between APD and the preceding diastolic interval is known as the electrical restitution properties ${ }^{68}$. The effect of $\beta$-adrenergic receptor stimulation on electrical restitution has been studied across the ventricular epicardium both in vitro ${ }^{24,25}$ and in vivo ${ }^{69}$, and transmurally in ventricular 
wedge preparations ${ }^{70}$ thanks to multielectrode arrays and optical mapping techniques. Sympathetic stimulation produces regional apex-base changes in restitution kinetics ${ }^{24,25,71}$, which are not seen in response to infusions of exogenous noradrenaline ${ }^{72}$, and arise from differences in regional innervation. This heterogeneity in recovery of excitability has the potential to be pro-arrhythmic. Focal epicardial application of noradrenaline causes triggered activity and computational modelling suggests that adrenergic-induced $\mathrm{Ca}^{2+}$ overload can activate the NCX current. This activation can drive more positive charge, pushing the membrane potential closer to the threshold for depolarization ${ }^{73}$. This idea that heterogeneity or gradients of activation are pro-arrhythmic is further supported by studies using optical mapping techniques in innervated, isolated, rabbit hearts ${ }^{74}$.

[H2] A sustaining substrate for re-entry and the sympathetic nervous system. Re-entry requires a central area of conduction block, which can be anatomical or functional, and also unidirectional conduction block or refractoriness at initiation, such that both waves of excitation around the area of central conduction block do not collide and extinguish each other. Re-entry, therefore, requires an appropriate substrate ${ }^{75}$. A substrate can be acquired during local ischaemia, which uncouples gap junctions and reduces the resting potential thereby preventing some fast $\mathrm{Na}^{+}$channels from recovering from the inactivated state, leading to slowed conduction ${ }^{76}$. Overtime, the substrate can become anatomically fixed, with thin, slow-conducting fibres of cardiomyocytes within the infarct scar that can promote re-entry ${ }^{77}$. This risk of re-entry is exacerbated by changes in the expression of membrane ion channels and gap junction proteins in the infarct border zone ${ }^{78}$, which can slow conduction. Structural abnormalities which may act as a substrate can also arise due to genetic conditions such as in arrhythmogenic right ventricular cardiomyopathy, hypertrophic cardiomyopathy and inherited dilated cardiomyopathy (particularly lamin A/C mutations) ${ }^{79}$. Substrates also have a dynamic component because electrophysiological properties can be influenced by hypoxia, haemodynamic, metabolic and ionic changes, drugs and autonomic tone. For example, whereas stimulation of stellate ganglia increases conduction velocity ${ }^{80}$, modulation of propagation after $\mathrm{Ml}$ is heterogeneous in the healed scar, with some regions having paradoxical conduction slowing, promoting an arrhythmogenic substrate in the peri-infarct region ${ }^{23}$.

The sympathetic innervation of an infarct border zone also undergoes substantial remodelling, with denervation occurring immediately after infarction, followed by subsequent reinnervation ${ }^{81}$. The exact contribution of sympathetic reinnervation in the genesis of arrhythmia in the infarct border zone is yet to be fully determined. Preventing denervation of the infarct border zone through protease inhibition does not seem to influence the risk of arrhythmia ${ }^{82}$, whereas preventing denervation by targeting neuronal protein tyrosine phosphatase receptor- $\sigma$ (PTP $\sigma)$ seems to reduce the risk of arrhythmia ${ }^{83}$. Chondroitin sulfate proteoglycans present in the infarct scar tissue prevent sympathetic reinnervation by binding to PTP $\sigma$, but 
absence or modulation of PTPo restores innervation ${ }^{83}$. However, knocking out the gene encoding PTPo can also be pro-angiogenic ${ }^{84}$ and improve revascularization. Interestingly, promoting reinnervation of the infract border zone through nerve growth factor (NGF) infusion in the stellate ganglia after MI results in an increased incidence of ventricular arrhythmia and sudden cardiac death ${ }^{85}$. In a dog model, NGF levels increased after $\mathrm{Ml}$, leading to heterogeneous hyperinnervation ${ }^{86}$. However, the interpretation of this observation might be more complex because NGF can influence the development of afferent neurons whose activity is stimulated by acute ischaemia ${ }^{87}$. Evidence suggests that the neuropathy associated with diabetes mellitus can also extend to cardiac afferent innervation and contribute to, and this arrhythmogenic neuropathy can be reversed by $\mathrm{Ngf}$ gene transfer ${ }^{89}$. The role of different neurotrophic factors in setting the balance between improved afferent sensing and worsening heterogeneous efferent innervation after MI deserves further study because it might reveal novel therapeutic opportunities.

Comparing these animal studies with clinical imaging studies using catecholamine analogues such as metaiodobenzylguanidine (ADMIRE-HF trial ${ }^{90}$ ) and $11 \mathrm{C}$-metahydroxyephedrine (PARAPET trial ${ }^{91}$ ) is interesting. The findings of these studies suggest that both the total volume of denervated myocardium and the volume of viable denervated myocardium after infarction predict ventricular arrhythmias and sudden cardiac death. These observations might be explained if excessive hyperinnervation or denervation produced similar electrophysiological effects and were equally pro-arrhythmic ${ }^{92}$. Regions of hyperinnervation were observed in explanted hearts from patients with a history of ventricular arrhythmia who received a heart transplant ${ }^{93}$; however, these regions were not the predominant phenotype in the border zone of the injured myocardium, but rather local findings at certain border zones, suggesting that a combination of denervation and hyperinnervation occurs. Alternatively, the border zone of the injured myocardium might be structurally hyperinnervated in terms of nerve density, but functionally behave as if denervated. Interpreting studies is further complicated by the observation that both $\beta$-adrenergic receptor supersensitivity ${ }^{83}$ and downregulation ${ }^{94}$ have been observed in the border zone of the injured myocardium. In ventricular wedge preparations from failing human hearts, $\beta_{2}$-adrenergic receptor stimulation seems to have a more prominent role when $\beta_{1}$-adrenergic receptors are downregulated, shortening the APD and increasing transmural heterogeneity ${ }^{70}$.

As a MI scar heals and chronic HF develops, changes in other inflammatory and neurohumoral pathways, including a range of growth factors and cytokines, can also become involved. These factors include members of the IL-6 family and leukaemia inhibitory factor ${ }^{10}$, which via gp130 signalling pathways can induce a phenotype switch in neurons from an adrenergic to a cholinergic phenotype. Around 2 weeks after $\mathrm{MI}$, acetylcholine levels in the infarct border zone transiently increase as sympathetic neurons start to express and release acetylcholine and noradrenaline ${ }^{95,96}$. In chronic HF, histological and molecular analysis of stellate ganglia from animal models ${ }^{97}$ and humans ${ }^{98}$ demonstrate increased staining for the synthetic enzymes of 
norepinephrine such as tyrosine hydroxylase as well as evidence of inflammation, neurochemical remodelling, oxidative stress, and satellite glial cell activation ${ }^{99}$. In a chronic MI model, increased expression of neuronal nitric oxide synthase (nNOS) has been observed in the ventral interventricular ganglionated plexus, dorsal root ganglia and stellate ganglia ${ }^{35}$. These changes might be a hallmark of early compensation involving nitric oxide (NO) pathways to modulate sympatho-vagal balance ${ }^{100}$.

[H2] Parasympathetic influence on ventricular arrhythmias. Although cholinergic innervation of the ventricles is relatively sparse compared with the ganglionic plexi in and around the atria, epicardial and endocardial ventricular innervation has been observed across a range of mammalian species ${ }^{101-104}$. Stimulation of the left cervical vagus nerve can decrease left ventricular contractility in pigs and humans ${ }^{105}$, although the sites of preganglionic nerve terminations in parasympathetic ganglia varies across species ${ }^{104,106,107}$. Of note, the cervical vagus nerve is composed of up to $70 \%$ afferent fibres ${ }^{108}$, which can also be recruited during stimulation. For example, if the vagus nerve is not transected, vagus nerve stimulation (VNS) can activate afferent fibres, with accompanying reflex inhibition of cardiac electrophysiological and haemodynamic effects ${ }^{109}$ The experimental approach, as well as stimulation parameters and patterns used, are therefore critical in determining the overall physiological response ${ }^{110}$.

Much has been written about the experimental basis and clinical implications of the role of the cardiac vagus nerve in the control of heart function ${ }^{111,112}$. The protective effect of the vagus nerve on the heart has been recognised since $1859^{113}$, and many studies have demonstrated that activation of the vagus nerve can prevent ventricular arrhythmia both in the normal heart and in the context of acute ischaemia and reperfusion ${ }^{114-118}$. Some of the most convincing evidence has come from studies by Peter Schwartz and colleagues. These researchers showed that in dogs with a healed MI, occlusion of the circumflex artery while the dogs were running on a treadmill triggered ventricular fibrillation (VF) in nearly two thirds of the animals ${ }^{47,119}$. The incidence of VF could be reduced by right VNS ${ }^{120}$ and the protective effect of VNS was abolished by administration of atropine, a blocker of muscarinic receptors.

[H2] Acute effect of cholinergic signalling on ventricular arrhythmias Depolarization of postganglionic vagal cholinergic neurons [G] causes the release of acetylcholine, which acts on muscarinic receptors. In sinoatrial node cells, activation of $M 2$ muscarinic acetylcholine receptors leads to hyperpolarization via $G_{k}$ stimulation of the acetylcholine-activated inward-rectifier $\mathrm{K}^{+}$current $\left(/_{\mathrm{KACh}}\right)$ and to a slowing in the rate of diastolic depolarization via $\mathrm{G}_{\mathrm{i}}$-dependent inhibition of adenylate cyclase, which reduces cAMP levels and the hyperpolarization-activated $\mathrm{Na}^{+}$inward current (or funny current; $I_{\mathrm{f}}$ ) and reduces PKA-dependent 
phosphorylation of L-type $\mathrm{Ca}^{2+}$ channels Overall, these effects produce an abrupt reduction in the heart rate $^{121}$. Heart rate reduction itself can be beneficial because it lowers the cardiac metabolic demand while improving diastolic coronary perfusion time and oxygen delivery. Some arrhythmogenic mechanisms, such as instability of intracellular $\mathrm{Ca}^{2+}$ handling that causes electrical alternans [G], are also more prevalent at high heart rates ${ }^{122}$. Several studies have demonstrated that the antiarrhythmic action of VNS can be blunted

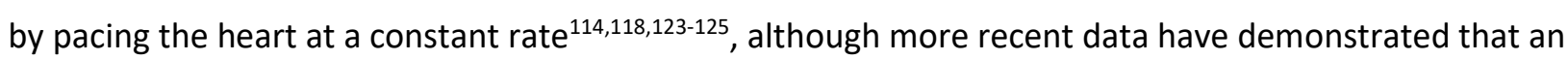
antifibrillatory effect of VNS still persists during cardiac pacing at a fixed heart rate ${ }^{126,127}$.

Stimulation of the M2muscarinic receptor in the ventricle is also coupled to $G_{i}$ and $G_{k}$ signalling cascades. $G_{i-}$ dependent reduction in $I_{\text {CaL }}$ together with decreases in SERCA2 activity (regulated by phospholamban) prevent $\mathrm{Ca}^{2+}$ overload and the generation of DADs $\mathrm{G}_{\mathrm{i}}$-dependent reduction in $/_{\mathrm{ks}}$ can also prolong the ventricular action potential and refractory period. In addition, the vagus nerve can have an indirect arrhythmogenic effect via 'accentuated antagonism' of adrenergic signalling. M2 Muscarinic receptor activation of $\mathrm{G}_{\mathrm{k}}$ leading to increased $I_{\mathrm{KACh}}$ is proposed to be the main mechanism mediating cholinergic accentuated antagonism of $\beta$-adrenergic receptors ${ }^{128}$. Other investigators have proposed that accentuated antagonism occurs at the levels of CAMP, whereby M2 muscarinic receptor stimulation of endothelial NO synthase (eNOS) ${ }^{129}$ can increase the activity of the CGMP-dependent phosphodiesterase PDE2A to reduce adrenergic-mediated cAMP signalling ${ }^{130}$. However, other studies demonstrate that accentuated antagonism of heart rate and contractility by the vagus nerve is preserved despite pharmacological inhibition of this pathway ${ }^{131}$ or gene knock out of Nos3, which encodes eNOS ${ }^{132}$.

Of note, voltage mapping experiments in isolated rabbit hearts demonstrate a prolongation of the ventricular refractory period, flattening of the APD restitution curve and a rise in the VF threshold in response to both left or right $\mathrm{VNS}^{133}$ or to cholinergic receptor stimulation ${ }^{127}$, in the absence of $\beta$-adrenergic receptor stimulation. Prolongation of the refractory period during increased vagal efferent activity is also observed independently of heart rate in clinical electrophysiological studies ${ }^{134}$.

NO generated by nNOS also has an important role in the antifibrillatory effect of cholinergic stimulation ${ }^{126}$. nNOS can alter parasympathetic neurotransmission in terms of vagal control of the heart rate. Pharmacological manipulation of NO signalling ${ }^{135,136}$ or knock out of Nos1 (which encodes nNOS) blunts the response to VNS but does not alter the bradycardia triggered by muscarinic receptor stimulation, indicating that the site of action of the nNOS-derived NO is pre-synaptic. Moreover, NO induces an increase in acetylcholine release in vagal neurons, mediated by augmenting cGMP levels, which inhibits PDE3, thereby leading to an increase in cAMP levels and PKA-dependent phosphorylation of $\mathrm{N}$-type $\mathrm{Ca}^{2+}$ channels ${ }^{131}$

In rabbits, the antifibrillatory effect of left or right VNS in vitro persisted despite atropine administration, but could be prevented by NOS inhibition ${ }^{126,137}$, suggesting that NO is released as a co-transmitter from 
parasympathetic efferent or afferent fibres. Whereas NOS inhibition prevented the effect of VNS on APD restitution, a shortening of the refractory period still persisted. By contrast, nNOS inhibition reduced the ventricular refractory period in rats with through silencing of dorsal vagal motor nucleus preganglionic neurons ${ }^{139}$.

Other studies show that the antiarrhythmic effects of cholinergic receptor stimulation are co-dependent on neuronal NO production and muscarinic receptor stimulation. In isolated rat hearts, an acetylcholine analogue prolonged APD, flattened the APD restitution curve and increased the VF threshold ${ }^{127}$. This increase in the VF threshold was abolished by specific and non-specific nNOS inhibition or by downstream inhibition of soluble guanylyl cyclase. An increase in NO metabolites was also observed, which was prevented by inhibition of nicotinic receptors on postganglionic cholinergic neurons. The antifibrillatory effect of both the acetylcholine analogue and a NO donor was completely abolished by atropine administration, suggesting that nNOS-derived NO modulates vagal neurotransmission ${ }^{136}$. The critical role of muscarinic receptors in the antiarrhythmic effect of VNS is also supported by recent studies of VNS in ischaemia-reperfusion injury ${ }^{140}$, as well as by classical in vivo studies that used atropine to block the antiarrhythmic effect of VNS $115,117,120,141,142$. The most likely mechanistic explanation for these observations is that nNOS-derived NO from parasympathetic ganglia facilitates acetylcholine release from sites of ganglionic projections. Whether $\mathrm{NO}$ also acts as a co-transmitter might depend on the density of innervation and the expression or activity of nNOS, given that NO is a highly diffusible and reactive signalling molecule ${ }^{143}$.

[H2] Chronic effect of cholinergic signalling on the arrhythmogenic substrate. A heterogeneous, fibrotic MI scar is an important substrate for the formation of re-entry and VT. Inflammatory pathways have a key role in fibrosis ${ }^{144}$, scar formation and hypertrophy ${ }^{145}$, and inflammatory mediators such as IL-1 can be directly arrhythmogenic ${ }^{146}$. VNS in rats during ischaemia-reperfusion reduces the infarct size, the number of inflammatory cells in the area at risk of injury and the levels of circulating inflammatory cytokines via a nicotinic receptor pathway ${ }^{147}$. Chronic VNS in a dog model of HF also normalizes the levels of IL-6 and TNF ${ }^{148}$, and reduces plasma levels of angiotensin II ${ }^{149}$, a potent pro-fibrotic mediator. Conduction velocity through scar tissue and in the normal myocardium critically depends on the expression and function of gap junction proteins. After MI, chronic VNS preserved connexin 43 phosphorylation and reduced the prevalence of spontaneous VT in rats ${ }^{150}$, and normalized connexin 43 levels in a dog model ${ }^{151}$. The chronic effects of VNS on myocardial substrate remodelling might have an important role in its antiarrhythmic effect.

Cardiac gene transfer of Nos1 targeted at the right atrium via a pericardial approach has been tested in a small animal model and shown to increase vagal nerve activity and reduce heart rate ${ }^{152}$ and mortality after $\mathrm{MI}^{5}$. However, targeting cholinergic ganglia in the ventricle with the use of this approach as an 
antiarrhythmic therapy has not been tested yet, although gene transfer directly into the cervical vagus nerve can increase cardiac baroreflex sensitivity in pigs ${ }^{153}$. Of interest, exercise training improves baroreflex control of heart rate and reduces susceptibility to arrhythmia ${ }^{154}$. Exercise training can also upregulate cardiac vagal nNOS activity, promote vagal transmission ${ }^{155}$ and also decrease sympathetic responses ${ }^{156}$, suggesting that cholinergic signalling is part of an endogenous protective pathway associated with the beneficial effects of physical training.

\section{[H1] Neuromodulation in ventricular arrhythmia}

Primary percutaneous coronary intervention and improved medical therapy have revolutionised the treatment of patients with $\mathrm{Ml}$; however, subsequent sudden cardiac death remains an important clinical problem. Enhanced sympathetic nerve activity is typical in patients with previous $\mathrm{MI}$ or with $\mathrm{HF}$, and is a key contributor to the increase in arrhythmogenicity observed in these patients. These patients can present with VT that is recurrent or incessant, resulting in haemodynamic instability and high mortality and in patients with an implantable cardioverter-defibrillator (ICD), requiring multiple ICD shocks ${ }^{162}$.

The potential for VNS to provide an effective neuromodulatory approach for the treatment of ventricular arrhythmia is well founded in mechanistic experimental studies, as described in the previous section, but has yet to be convincingly translated into clinical practice. Clinical studies on VNS for the treatment of advanced $\mathrm{HF}$ have been disappointing and have not targeted reduction of ventricular arrhythmia as an end point. Pharmacological approaches for the treatment of ventricular arrhythmia in HF, such as cholinesterase inhibition ${ }^{157}$, also have limitations. Therefore, the sympatho-adrenal axis has been the principal target for drug treatment with $\beta$-blockers and angiotensin-converting enzyme inhibitors in patients with $\mathrm{MI}$ or with $\mathrm{HF}$, with both drug therapies inducing a significant reduction in morbidity and mortality ${ }^{158-161}$.

Current secondary prevention VT management strategies include the use of antiarrhythmic drugs and catheter ablation ${ }^{163}$, but durable freedom from recurrent VT remains suboptimal ${ }^{164}$. While repeat ablation procedures remain as a treatment option for $\mathrm{VT}$, additional therapies targeting the sympatho-adrenal neural axis have shown promise and have moved from bench to bedside (Figure 1). Experimental studies have supported this paradigm shift in therapeutic approach.

[H2] Cardiac sympathetic denervation. The anterior wall of the left ventricle is innervated by bilateral stellate ganglia and their stimulation results in noradrenaline release and electrophysiological changes that increase the risk of ventricular arrhythmia ${ }^{165,166}$. The stellate ganglia in patients with cardiomyopathy and ventricular arrhythmia are characterized by hypertrophy, inflammation, remodelling and oxidative stress ${ }^{98}$. The 
remodelling process after myocardial injury takes place at multiple levels of the cardiac neural axis and also affects the intrinsic cardiac nervous system (ICNS), with heterogeneity in the afferent signal from the scar and border zone regions ${ }^{36}$. The deleterious effects of cardiac neural axis remodelling on cardiac electrophysiology in a pig model could be reversed with bilateral stellectomy (a procedure known in patients as cardiac sympathetic denervation, CSD), resulting in a reduction in VT inducibility and a small correction in activation recovery interval (a surrogate for APD) ${ }^{167}$.

The clinical efficacy of CSD was first demonstrated in the context of LQTS ${ }^{168,169}$ and catecholaminergic polymorphic $\mathrm{VT}^{170}$. In the largest study in patients with LQTS who were undergoing left CSD, >50\% of previously symptomatic patients became asymptomatic and in the remaining patients, the annual rate of cardiac events decreased by up to $91 \%{ }^{171}$. In patients with $\mathrm{HF}$ and with refractory ventricular arrhythmia that did not respond to catheter ablation, a large, contemporary study reported the efficacy of left or bilateral $\mathrm{CSD}^{172,173}$. This approach resulted in a $>80 \%$ reduction in the number of ICD shocks after the procedure, with bilateral CSD being more effective than left CSD alone (Figure $5 \mathrm{c}$ ).

[H2] Thoracic epidural anaesthesia. Thoracic epidural anaesthesia (TEA) has also demonstrated therapeutic efficacy in this patient population, and can be performed as an emergency procedure at the bedside ${ }^{174}$. The mechanisms of TEA have been studied in dog models ${ }^{175}$ and involve attenuation of afferent and efferent cardiac neurotransmission with prolongation of the myocardial refractory period ${ }^{176}$. With this approach, patients can be safely bridged to more definitive therapies. The largest, multicentre clinical study on TEA included 11 patients with VT storm [G] ${ }^{177}$. All patients tolerated the TEA without haemodynamic compromise, with $>50 \%$ of patients experiencing a complete or partial response to the therapy. One of the major limitations of TEA is that this approach cannot be performed with concomitant use of antiplatelet medications or anticoagulants; therefore, an alternative percutaneous strategy for patients requiring antithrombotic therapy is ultrasound or fluoroscopy-guided, cervico-thoracic stellate ganglion block. A metaanalysis of studies on stellate ganglion block demonstrated a significant decrease in ventricular arrhythmias and the number of ICD shocks after the procedure that was independent of the underlying aetiology ${ }^{178}$.

[H2] Renal denervation. Mechanistic studies have shown that renal denervation can prevent noradrenaline spillover, which is extenuated by abnormal sympathetic afferent activity ${ }^{179}$ and influences ventricular electrophysiology ${ }^{180,181}$. Small-scale clinical trials have reported that bilateral renal denervation is an effective therapy for refractory ventricular arrhythmia ${ }^{182-184}$. This approach requires optimization in terms of denervation site and energy delivery before larger studies are undertaken to confirm its efficacy, in view of the disappointing results of the SYMPLICITY HTN-3 trial of renal denervation in drug resistant hypertension ${ }^{185}$, although it is worth noting that more recent randomized, sham controlled trials in patients either taking (SPYRAL HTN-ON trial) or not taking antihypertensive medication (SPYRAL HTN-OFF MED trial ${ }^{186}$ and RADIANCE SOLO trial) seem more promising. 
[H2] Approaches at experimental stages targeting the sympatho-adrenal neural axis. Other emerging targets for neuromodulation of the sympatho-adrenal neural axis include distal ligament of Marshall ablation and optogenetic manipulation of stellate ganglia physiology. The distal ligament of Marshall serves as a conduit between the left stellate ganglia and the ventricular myocardium. In a dog model of MI, ablation at this site prevented ventricular arrhythmia ${ }^{187}$. A study in dogs published in 2017 utilized an adenovirus associated vector to deliver to stellate ganglia the light-activated proton pump archaerhodopsin $\mathrm{T}$ (ArchT), which produces inhibitory currents when stimulated with green LED light ${ }^{188}$. The vector demonstrated specificity for the stellate ganglia and stimulation with light suppressed sympathetic activity, as evidenced by prolongation of ventricular APD and effective refractory period, improved heart rate variability and protection against ischaemia-induced ventricular arrhythmia.

Sympathetic neuromodulation can also be achieved at sites far removed from the cardio-renal axis. Deep brain stimulation of the periaqueductal grey is used for the treatment of chronic pain, but can alter the blood pressure depending on location of the stimulation electrode ${ }^{189,190}$, heart rate variability ${ }^{190}$ and baroreflex sensitivity by influencing sympathetic outflow ${ }^{191}$. Spinal cord stimulation at T1-T3 has also been shown to reduce cardiac sympathetic drive, and continuous spinal cord stimulation improved symptoms in a small group of patients with severe symptomatic $\mathrm{HF}^{192}$. Furthermore, an animal model of spinal cord stimulation demonstrated suppression of left stellate ganglia activity and post-MI ventricular arrhythmia ${ }^{193}$. However, the randomized, single-blind DEFEAT-HF trial ${ }^{194}$ did not provide evidence of any meaningful change in clinical outcomes in patients with HF.

[H2] Vagus nerve stimulation. As discussed above, direct cervical VNS has demonstrated substantial promise in animal models of post-MI ventricular arrhythmia, which might be related to the observation that acetylcholine levels and parasympathetic neuronal pathways are preserved in the infarct border zone, enabling electrical stabilization after VNS ${ }^{195}$. However, after a promising proof-of-concept study ${ }^{198}$, subsequent clinical trials of VNS in advanced HF (including NECTAR-HF and INOVATE-HF) had disappointing results ${ }^{196,197}$. Although arrhythmia end points were not evaluated in these trials, HF symptoms did not improve. The ANTHEM-HF study ${ }^{199200}$ compared right or left VNS in a small group of patients and demonstrated improvements in symptoms and echocardiographic parameters at both 6 months and 12 months of follow up compared with baseline, although the trial did not include a control group. A substudy of Holter recordings from this study also demonstrated a reduction in the incidence of T-wave alternans and non-sustained VT, as well as favourable effects on heart rate turbulence, a marker of baroreceptor function ${ }^{201}$. On the back of these results, a large, randomized, controlled trial of right VNS with the use of the same system is now underway (ANTHEM-HF PIVOTAL trial, NCT03425422). Of note, these trials used a variety of stimulation parameters and techniques, which might influence the efficacy of the approach ${ }^{110,202}$. How this and other aspects of device and trial design might have led to neuromodulation being 'lost in 
translation' have been discussed extensively ${ }^{203}$. Given the scientific basis for VNS and the opportunities to improve stimulation parameters, programming and hardware, there is scope to be optimistic for this approach, especially given the long record of safety and efficacy of VNS in the treatment of $>100,000$ patients with drug-resistant epilepsy.

\section{[H1] The ANS and atrial fibrillation}

Atrial fibrillation (AF) is the most common pathological cardiac arrhythmia, and is associated with significant morbidity and mortality. AF produces a cascade of structural and electrical remodelling in the atria including chamber dilatation and wall fibrosis ${ }^{204}$ such that further AF becomes increasingly more likely and restoration of sinus rhythm more difficult. This phenomenon is encompassed by the well-known adage that 'AF begets $\mathrm{AF}^{205}$. The seminal work of Haissaguerre and colleagues defined the role of rapid firing from the pulmonary veins in initiating paroxysmal $\mathrm{AF}^{206}$. Of note, the pulmonary veins contain a variety of myocytes capable of generating ectopic activity, which can then reach the atria and induce $\mathrm{AF}^{207}$. The pulmonary veins might also provide a non-excitable gap to support re-entry, and are also highly innervated ${ }^{208}$. The atrial ICNS is an extensive, interconnected epicardial network of ganglionic plexi, nerve axons and interconnecting neurons. These structures are embedded within the epicardial fat pads, except the ligament of Marshall, which runs between the left atrial appendage (LAA) and left pulmonary vein antrum. These structures vary in size from those containing a few neurons to some with $>400$ neurons ${ }^{209,210}$.

The vagal nerve influence on AF has been known historically and VNS or acetylcholine administration can lead to $A F^{211}$. Furthermore, some forms of paroxysmal $A F$ are related to elevated vagal tone ${ }^{212}$. Of note, exercise training has been associated with an increased risk of $A F^{213}$, although exercise has a variety of beneficial effects on overall cardiovascular health. This relationship seems to become stronger with age ${ }^{214}$ and confers an overall fivefold higher risk of $\mathrm{AF}$ in athletes compared with sedentary individuals ${ }^{215}$. AF in athletes also occurs during periods of high vagal tone, such as at night, at cessation of exercise and in the post-prandial period ${ }^{216}$.

The electrophysiological effects underpinning these observations include spatially heterogeneous atrial refractoriness ${ }^{217,218}$, and shortening of atrial propagation wavelength ${ }^{219}$ with cholinergic activation. These effects might be mediated at least in part by increasing $I_{\text {KAch }}$ in a spatially heterogeneous manner, thereby promoting re-entry. Direct recording of stellate ganglia and vagus nerve activity in animals enables correlation with temporal changes in nerve activity and AF initiation ${ }^{220}$ Simultaneous activation of both branches of the ANS might be required to initiate AF. This requirement would be unsurprising given that the effects of muscarinic receptor activation are exacerbated during background $\beta$-adrenergic receptor 
stimulation, via accentuated antagonism. Direct stimulation of ganglionic plexi associated with the left atrial fat pads can lower the threshold for induction of $A F$ or precipitate paroxysms of $A F^{221}$. Acetylcholine injection into ganglionic plexi results in firing from adjacent pulmonary vein ${ }^{222}$ and sustained AF. Furthermore, the cellular electrophysiology of the pulmonary vein muscular sleeve has enhanced sensitivity to autonomic stimuli, which allows a shorter APD than in the surrounding atrial myocardium ${ }^{223,224}$.

Experimental studies with autonomic stimulation of isolated pulmonary veins demonstrate induction of early afterdepolarizations and triggered activity similar to those seen in patients with paroxysmal $\mathrm{AF}^{223}$. Highfrequency stimulation in the vein of Marshall can also trigger AF and atrioventricular nodal conduction slowing, demonstrating a communication between ganglionic plexi in the vein of Marshall and the right inferior ganglionic plexi (which controls the atrioventricular node). Such a response was eliminated after ethanol infusion in the vein of Marshall225, which produced regional parasympathetic denervation ${ }^{226}$. Targeting the ligament of Marshall has traditionally been performed during epicardial surgical AF ablation (the maze procedure, also known as Cox maze III), and percutaneous ethanol infusion in the vein of Marshall is currently being assessed in a randomized clinical trial ${ }^{227}$.

Although electrical and structural remodelling of the atria in AF is widely appreciated, comparatively little is known about neural remodelling and how it may contribute to AF. A heterogeneous increase in atrial sympathetic nerve density has been observed both in animal models of $A F^{228,229}$ and in patients with $A F$ who were undergoing cardiac surgery ${ }^{230}$ Low-level stimulation of the cervical vagus nerve, either directly or transcutaneously via the tragus, is thought to silence ganglionic plexi, reduce systemic markers of inflammation and AF inducibility ${ }^{231}$ and decrease the occurrence of paroxysmal AF and post-operative AF after cardiac surgery ${ }^{232}$. Inflammation has an important role in the pathogenesis of AF and might have a role in neural, myocyte and fibrotic remodelling, whereby inflammation and remodelling can perpetuate each other and maintain $\mathrm{AF}^{231}$. Ganglionic plexi and the ICNS function independently or in response to higher levels of the cardiac neural axis. Ganglionic plexi and the ICNS can form a final effector pathway in the autonomic, pro-fibrillatory influence on the atria, which makes these structures an attractive, anatomically accessible target for therapy in patients with AF. However, the basic physiology of this complex neural network is still being elucidated.

\section{[H1] Neuromodulation in atrial fibrillation}

The current methodology for percutaneous catheter ablation in AF involves wide, circumferential pulmonary vein isolation (PVI) with either radiofrequency or cryoablation. The current approach for antral PVI inevitably also targets the anatomical locations of the ganglionic plexi, and the physiological effects include 
bradycardia during ablation ${ }^{233}$ and alterations in heart rate variability in the long term ${ }^{234}$. A single, left atrial ganglionic plexus is thought to innervate one of the pulmonary veins as well the surrounding atrial myocardium ${ }^{209,210}$ (Figure 4a). Autonomic denervation is seen frequently after $\mathrm{PVI}^{235-237}$ and observational studies have reported a decrease in AF recurrence when this denervation occurs ${ }^{238-240}$. Complete PVI might not be required to maintain sinus rhythm, also supporting the observations of autonomic denervation ${ }^{238,241-}$ ${ }^{243}$. The conventional lesion set after PVI transects the anatomical location of three of the major ganglionic plexi sites; therefore, interruptions of these projections might contribute to procedural success ${ }^{244}$. However, the specific neural elements that are responsible for the therapeutic effect of PVI remain to be determined.

Small, randomized, controlled trials have supported the role of ganglionic plexi ablation in addition to $\mathrm{PVI}^{239,240}$ or as an isolated strategy ${ }^{245}$ for the treatment of AF. Addition of ganglionic plexi ablation improved success rates by $25 \%$, whereas ganglionated plexi ablation alone in paroxysmal AF or persistent AF was successful in $71-86 \%$ of the patients. The largest randomized trial on the effect of ganglionated plexi ablation combined with PVI in 242 patients with paroxysmal AF demonstrated a significant improvement in AF and atrial tachycardia free survival in the group receiving ganglionated plexi ablation and PVI compared with the groups receiving PVI alone or ganglionated plexi ablation alone ${ }^{246}$. A similar approach in patients with persistent AF showed that PVI plus ganglionated plexi ablation was superior to PVI plus linear ablation, with the additional benefit of a decrease in left atrial tachycardias ${ }^{247}$. However, in patients with drugrefractory, long-standing, persistent $\mathrm{AF}$, ganglionated plexi ablation alone resulted in worse outcomes than $\mathrm{PVI}^{248}$.

Some data correlate the areas of endocardial complex fractionated atrial electrograms (CFAEs) in AF with the presence of ganglionated plexi ${ }^{299-251}$, although imaging accurately the anatomical location of ganglionated plexi during an ablation procedure is currently not possible. The results of the large, randomized, STAR-AF II trial ${ }^{252}$ assessing PVI alone, PVI plus left atrial linear ablation and PVI plus ablation of CFAE sites for the treatment of persistent AF should also be considered. In this study, which has influenced clinical practice worldwide, PVI alone was superior to the other approaches. Ganglionated plexi activity might be most relevant in paroxysmal $\mathrm{AF}$, while becoming less important with progression to persistent $\mathrm{AF}$ and the development of atrial remodelling and fibrosis ${ }^{231}$.

Another approach to localize the ICNS or ganglionated plexi is based on the response to endocardial high frequency $(2 \mathrm{~Hz})$ stimulation while looking for inhibition of atrioventricular nodal conduction or increases in the $\mathrm{R}-\mathrm{R}$ interval by $50 \%$ during $\mathrm{AF}^{240}$. Although a single-centre study reported improvements in outcome with the use of high frequency stimulation to target ganglionated plexi ablation, more ablation was undertaken in this group, which might have influenced these results. Experimental observations in animals ${ }^{253}$ and humans ${ }^{254}$ have suggested that targeting ganglionated plexi with inputs to pulmonary veins might produce better results than high frequency stimulation. The electrophysiological approach based on identification of 
vagal responses also lack sensitivity ${ }^{231}$. Atrial ganglionated plexi contain a complex network of efferent, afferent and inter-neuronal populations expressing a variety of neurotransmitters. Disruption of atrial cholinergic neurons can also have a detrimental influence on ventricular electrophysiology because cholinergic neurons run alongside sympathetic fibres from the atria to the ventricles ${ }^{255}$, particularly after acute $\mathrm{Ml}^{256}$.

In addition, a complex series of interactions exists between the extrinsic cardiac nervous system (ECNS) and the $\mathrm{ICNS}^{257}$, which might enable the exploration of alternative neuromodulation therapies. The ganglionated plexi integrate autonomic inputs with multiple interconnections with a final common pathway to the sinus and atrioventricular nodes via the right anterior and inferior ganglionated plexi. Ablation of ganglionated plexi between the ECNS and ICNS leads to an increase in AF burden, suggesting the presence of inhibitory effect to preserve sinus rhythm ${ }^{258}$. The reduction in vagal tone with ageing might, therefore, contribute to loss of this tonic suppression of ganglionated plexi activity and the corresponding increase in AF prevalence ${ }^{259}$. This interaction has been translated clinically into bioelectronic neuromodulation with low level VNS, a technique that has shown efficacy in epilepsy ${ }^{260}$ and in early clinical trials in $\mathrm{HF}^{198}$. Applying low level VNS without inducing bradycardia influenced downstream atrial electrophysiology, with an increase in the effective refractory period in the atrium and pulmonary veins, suppression of AF inducibility and a decrease in the duration of acetylcholine-induced $\mathrm{AF}^{261,262}$.

Direct recordings of ganglionated plexi activity during VNS demonstrate a decrease in the frequency and amplitude of discharges ${ }^{262}$. Surgical VNS applied extra-epicardially, rostral to the heart, has been studied in a randomized control trial in patients undergoing cardiac surgery ${ }^{263}$. Stimulation below the threshold for bradycardia resulted in a significant reduction in post-operative $\mathrm{AF}$ ( $12 \%$ versus $36 \%$ ) and the levels of circulating inflammatory cytokines. Interestingly, although VNS was applied only during the intensive care period after cardiac surgery (72 h), efficacy of AF suppression was maintained during the 1 month of followup. This memory effect of VNS has been previously reported ${ }^{31,264}$ and might indicate future implications for the application of novel neuromodulatory therapies. Surgical VNS is feasible in the context of patients undergoing cardiac surgery, but might not be applicable to the large number of patients with AF.

Low-level tragus stimulation (LLTS) targets the auricular branch of the vagus nerve and has been shown to result in brainstem evoked potentials [G] in humans ${ }^{265}$ and to suppress pacing-induced AF in experimental model ${ }^{266}$. LLTS restored normal anterior right ganglionated plexi activity following rapid pacing ${ }^{266}$. In patients with paroxysmal AF referred for ablation, LLTS decreased rapid-pacing-induced AF duration and plasma TNF levels ${ }^{232}$. Further studies are required to evaluate these and other novel approaches for the prevention and treatment of $A F$, including botulinum toxin injection into epicardial fat pads ${ }^{267}$ and renal denervation ${ }^{268}$. Of note, in a study in $>700$ patients receiving orthotopic heart transplants, in which the heart 
is completely decentralised, AF was only observed after the post-operative recovery period in the presence of rejection or vasculopathy ${ }^{269}$.

\section{[H1] Discovery of novel neuronal targets}

With the advent of high throughput sequencing, RNA sequencing provides the opportunity to identify key transcripts (for hypothesis testing) that might encode proteins involved in abnormal neurotransmission. This technique can be applied to whole tissue but can also be performed at a single cell level, making RNA sequencing ideally suited for studying neural remodelling of autonomic efferent and afferent nerves once isolated. For example, emerging evidence suggests that in stellate ganglia from rats with a sympathetic phenotype of hyperactivity, a significant number of differentially expressed transcripts compared with healthy rats are linked to impaired regulation of intracellular $\mathrm{Ca}^{2+}$ homeostasis and exocytosis ${ }^{270,271}$ (Figure 6). Moreover, these transcripts are conserved in human stellate neurons, thereby providing wider physiological context. Bardsley et al. reported differential expression of several transcripts that were validated using quantitative reverse transcription PCR (qRT-PCR) ${ }^{270}$. Several gene ontology groups were identified with network and enrichment analyses. The identified gene ontology families and functional pathways included transcripts related to extracellular ligand-gated ion channel activity, in particular glutamatergic and dopaminergic signalling pathways linked to modulating $\mathrm{Ca}^{2+}$ balance. PDE activity was also altered ${ }^{270}$, supporting previous reports indicating that impaired cyclic nucleotide signalling is coupled to sympathetic dysautonomia ${ }^{272}$.

Although changes in transcript levels do not directly equate to changes in protein levels, nor do they indicate protein-protein interactions, transcriptome changes nevertheless provide a framework to test the functional role of lead candidate genes in the regulation of physiological processes. For example, cells host several isoforms of PDEs, enzymes with dual-specificity for CAMP and cGMP that are involved in maintaining the balance of CAMP and CGMP levels. In stellate ganglia from rats with sympathetic hyperactivity, expression of $P d e 2 a$ and Pde11a was decreased whereas expression of $P d e 6 b$ was increased compared with stellate ganglia from normal rats. However, closer examination in diseased rats and human stellate tissue reveals that PDE2A protein and activity levels are increased in conditions of sympathetic hyperactivity ${ }^{273}$. Moreover, if PDE2A is directly up-regulated by overexpression with an adenovirus in healthy neurons, agonist-activated cGMP levels decrease due to increased PDE2A hydrolytic activity, thereby mimicking the diseased state. The decreased cGMP levels are associated with increased $\mathrm{Ca}^{2+}$ currents and $\mathrm{Ca}^{2+}$ transients, leading to greater release of neuronal noradrenaline ${ }^{274}$. Interestingly, in diseased neurons, pharmacological inhibition of PDE2A or gene transfer of the dominant negative form of PDE2A restores the inhibitory action of receptor-coupled cGMP activators to decrease intracellular $\mathrm{Ca}^{2+}$ and neurotransmission ${ }^{273}$. 
What is not fully established is whether the identified transcripts associated with impaired sympathetic function are fully conserved across mammalian genomes. Most of the lead candidate transcripts identified in the study by Bardsley et al. ${ }^{270}$ are present in the mouse stellate ganglia and, interestingly, a significant sex difference in expression levels has been observed ${ }^{275}$, which might need to be considered when studying other species. Although transcriptomes provide a molecular 'road map' for further studies, transcriptomics only provides a statistical association to promote a direction of investigation. Validation of the transcript at the protein level and, more importantly, showing that proteins encode function (physiology) and are conserved in human tissue, will be essential to establish target discovery and clinical utility.

Even though the use of transcriptomics is 'hypothesis neutral', this technique can nevertheless reveal surprising associations previously missed with the use of conventional pharmacological approaches. Bardsley et al. observed low-level expression of genes encoding pre-synaptic $\beta$-adrenergic receptors in the rat and showed with the use of qRT-PCR that these receptors were conserved in human stellate neurons ${ }^{40}$. Early studies in animal models had reported the presence of $\beta$-adrenergic receptors in postganglionic sympathetic neurons ${ }^{276}$ and suggested a possible role for these receptors in the regulation of noradrenaline release during nerve stimulation, the so called 'adrenaline hypothesis' of hypertension. However, the presence of these receptors in human tissue and the precise signalling pathway underpinning this hypothesis was not established until recently. Using a combination of RNA sequencing, immunocytochemistry, Forster resonance energy transfer imaging to assess CAMP-PKA activity, and measurements of intracellular $\mathrm{Ca}^{2+}$ levels, Bardsley et al. identified a functional $\mathrm{Ca}^{2+}$-dependent exocytosis that was predominately activated via a $\beta_{2}$-adrenergic receptor stimulation of the cAMP-PKA pathway in diseased neurons only ${ }^{40}$ (Figure 7). Increases in the levels of phenylethanolamine-N-methyltransferase led to neurotransmitter switching in preference for the synthesis of adrenaline in rat and human stellate neurons ${ }^{40}$ (Figure 7). Of interest, the study also reported an increased evoked release of both noradrenaline and adrenaline in diseased neurons from pre-hypertensive rats, establishing evidence that catecholamine levels were raised before the overt signs of hypertension and adding weight to the model that pre-synaptic release of noradrenaline and adrenaline might further drive sympathetic transmission through positive feedback stimulation of neuronal $\beta$-adrenergic receptors. Together with circulating catecholamines, neuronal release of catecholamines might be a powerful drive of cardiac postsynaptic excitability 277,278 (Figure 7).

The mechanism driving enhanced cardiac sympathetic transmission in cardiovascular disease is not fully established. What is clear is that the autonomic phenotype can precede the overt clinical signs of the disease ${ }^{279-281}$ and reside in both the peripheral and central part of the cardiac neural axis ${ }^{282}$. Several research groups have argued that oxidative stress is a key hallmark of sympathetic dysautonomia, and impairment of the NO-cGMP pathway has been implicated ${ }^{100}$. Under normal physiological conditions, NO-cGMP provides an inhibitory action on sympathetic transmission by activating cGMP-dependent stimulations of PDE2A to 
decrease cAMP-coupled phosphorylation of neuronal $\mathrm{Ca}^{2+}$ channels $^{283-285}$. Overexpression of Nos1 with a noradrenergic-specific promoter can rescue impaired NO-cGMP signalling and restore $\mathrm{Ca}^{2+}$-dependent exocytosis in a rat model of sympathetic hyperactivity $286,287$.

Another clue regarding the importance of the NO-cGMP pathway has come from the 'top down' approach of genome wide association studies (GWAS), rather than the 'bottom up' approach of transcriptomics. Modulation of the NO pathway is strongly influenced by the coupling of the nNOS adaptor protein CAPON (also known as NOS1AP) ${ }^{288}$. The potential importance of CAPON was first highlighted by the identification by GWAS of single nucleotide polymorphisms in NOS1AP associated with QT interval variation ${ }^{289}$ and with sudden cardiac death ${ }^{290}$. Polymorphisms in NOS1AP have also been identified as risk modifiers for arrhythmic events and sudden cardiac death in patients with LQTS type $1^{291}$. Overexpression of NOS1AP in cardiomyocytes led to shortening of the QT interval via a putative pathway in which CAPON-nNOS induced a cGMP-dependent inhibition of $I_{\text {Cat }}$ and activation of $I_{\mathrm{Kr}}$, causing a faster repolarization of the APD that resulted in restoration of the QT interval ${ }^{292}$. Of interest, one of the major triggers of sudden death in patients with certain types of LQTS is a sympathetic surge that is experienced during strenuous exercise and psychological stress ${ }^{293}$. CAPON was first discovered in the brain ${ }^{294}$, but is also present in peripheral autonomic ganglia ${ }^{288}$. Overexpression of this adaptor protein in sympathetic neurons decreases the $\mathrm{N}$-type $\mathrm{Ca}^{2+}$ current $\left(I_{\mathrm{CaN}}\right)$, the $\mathrm{Ca}^{2+}$ transient and subsequent neurotransmission via a cGMP-dependent pathway ${ }^{288}$. The functional significance of this pathway in modulating arrhythmia is not firmly established, but mutations in CAPON might facilitate abnormal sympathetic transmission and trigger afterdepolarizations (Figure 8).

\section{[H1] Conclusions}

Returning to the three-level neural hierarchical model of the ANS shown in Figure $2 b$, and the feedback loops that exist within and between levels, several things become clear. First, this model is an oversimplification of a complex neural network, and how the ANS is remodelled with disease is poorly understood. Moreover, the tools we have to interact with the system, in particular neural stimulation, surgical resection and ablation, are also crude. None of these interventions solely target efferent or afferent neurons from a single branch of the ANS. That said, we remain optimistic about the research community's ability to unravel this complexity. Techniques that have advanced neuroscience in the last decade, such as optogenetics, genetic manipulation, gene editing and CLARITY [G], combined with more traditional neurophysiological techniques are likely to make great inroads. However, prematurely racing towards clinical trials with little understanding of what we are doing risks damaging the entire field. We need to be very careful that we do not make the same mistakes seen in other medical fields throughout history. It 
would be a shame if intervention for arrhythmia on the 'heart's little brain' became for cardiology what lobotomy for schizophrenia became for neurosurgery.

Of note, some of the more successful interventions to date for ventricular arrhythmias target the stellate ganglia. The stellate ganglion is at least a tractable target, in that it is an important relay station for cardiac afferents and contains the cell bodies of mainly efferent post-ganglionic neurons communicating directly with the myocardium. The stellate ganglion is also easily accessible, at least when compared with the brainstem and other level 1 structures in the neural hierarchy. When assessing interventions (particularly stimulation parameters and patterns), these interventions should be systematically and thoroughly tested in representative animal models of disease before trialling in humans.

It should also be considered that patients will already be receiving the current standard of care in terms of pharmacotherapy. This therapy can include $\beta$-blockers and medications targeting the renin-angiotensinaldosterone system, which also influence the ANS. Therefore, these medications are likely to influence the efficacy of approaches to neuromodulation. Use of $\beta$-blockers, for example, might uncover a more prominent role for other adrenergic receptors or indeed co-transmitter receptors in the ongoing disease process. We suggest that new therapies are also tested in animal disease models receiving the same therapeutic treatment as patients with the disease to see whether the treatment influences the effectiveness of the new approach. The influence of sex differences should also be considered.

Although cardiac sympathetic denervation has shown significant clinical utility with a low complication rate, opportunities exist to refine our manipulation of this site (Figure 5). In this context, we feel that transcriptomics and proteomics of the stellate ganglia, and other neuronal populations might help to identify how the ANS remodels during disease, and in so doing, provide a molecular framework and road map to test the functional significance of these pathways. This approach might also, to some extent, help to validate how close different animal models are to their human disease counterparts, and potentially to identify new therapeutic targets. Finally, being able to differentiate human stem cells into cardiac cells and sympathetic neurons from patients with genetic autonomic phenotypes, and then study these cells in vitro, might aid us in understanding human pathophysiology.

\section{References}

1 Samuels, M. A. The brain-heart connection. Circulation 116, 77-84, doi:10.1161/CIRCULATIONAHA.106.678995 (2007).

2 Esler, M. Heart and mind: psychogenic cardiovascular disease. $J$ Hypertens 27, 692-695, doi:10.1097/HJH.0b013e328324f72b (2009).

3 Armour, J. A. Potential clinical relevance of the 'little brain' on the mammalian heart. Exp Physiol 93, 165-176, doi:10.1113/expphysiol.2007.041178 (2008). 
Krul, S. P. J. et al. Treatment of Atrial and Ventricular Arrhythmias Through Autonomic Modulation. JACC Clin Electrophysiol 1, 496-508, doi:10.1016/j.jacep.2015.09.013 (2015).

Dawson, T. A. et al. Cardiac cholinergic NO-cGMP signaling following acute myocardial infarction and nNOS gene transfer. Am J Physiol Heart Circ Physiol 295, H990-H998, doi:00492.2008 [pii] 10.1152/ajpheart.00492.2008 (2008).

Shen, M. J. \& Zipes, D. P. Role of the autonomic nervous system in modulating cardiac arrhythmias. Circ Res 114, 1004-1021, doi:10.1161/CIRCRESAHA.113.302549 (2014). Schwartz, P. J. Cardiac sympathetic denervation to prevent life-threatening arrhythmias. Nat Rev Cardiol 11, 346-353, doi:10.1038/nrcardio.2014.19 (2014). Ardell, J. L. et al. Translational Neurocardiology: preclinical models and cardioneural integrative aspects. J Physiol, doi:10.1113/JP271869 (2016). therapeutics. J Physiol, doi:10.1113/JP271870 (2016). Habecker, B. A. et al. Molecular and cellular neurocardiology: Development, cellular and molecular adaptations to heart disease. J Physiol, doi:10.1113/JP271840 (2016).

11 Herring, N. \& Paterson, D. J. Levick's introduction to cardiovascular physiology. Sixth edition. edn, (CRC Press, 2018).

12 Ardell, J. L., Cardinal, R., Vermeulen, M. \& Armour, J. A. Dorsal spinal cord stimulation obtunds the capacity of intrathoracic extracardiac neurons to transduce myocardial ischemia. Am J Physiol Regul Integr Comp Physiol 297, R470-477, doi:10.1152/ajpregu.90821.2008 (2009). Armour, J. A. Activity of in situ stellate ganglion neurons of dogs recorded extracellularly. Can J Physiol Pharmacol 64, 101-111 (1986).

14 Paintal, A. S. Vagal Afferent Fibres. Ergeb Physiol 52, 74-156 (1963).

15 Malliani, A., Recordati, G. \& Schwartz, P. J. Nervous activity of afferent cardiac sympathetic fibres with atrial and ventricular endings. J Physiol 229, 457-469 (1973).

16 Fukuda, K., Kanazawa, H., Aizawa, Y., Ardell, J. L. \& Shivkumar, K. Cardiac innervation and sudden cardiac death. Circ Res 116, 2005-2019, doi:10.1161/CIRCRESAHA.116.304679 (2015).

17 Vaseghi, M. \& Shivkumar, K. The role of the autonomic nervous system in sudden cardiac death. Prog Cardiovasc Dis 50, 404-419, doi:10.1016/j.pcad.2008.01.003 (2008). Rubart, M. \& Zipes, D. P. Mechanisms of sudden cardiac death. J Clin Invest 115, 2305-2315, doi:10.1172/JCI26381 (2005).

19 Rutherford, S. L., Trew, M. L., Sands, G. B., LeGrice, I. J. \& Smaill, B. H. High-resolution 3-dimensional reconstruction of the infarct border zone: impact of structural remodeling on electrical activation. Circ Res 111, 301-311, doi:10.1161/CIRCRESAHA.111.260943 (2012). Wit, A. L. et al. Electrophysiologic mapping to determine the mechanism of experimental ventricular tachycardia initiated by premature impulses. Experimental approach and initial results demonstrating reentrant excitation. Am J Cardiol 49, 166-185 (1982).

21 Dillon, S. M., Allessie, M. A., Ursell, P. C. \& Wit, A. L. Influences of anisotropic tissue structure on reentrant circuits in the epicardial border zone of subacute canine infarcts. Circ Res 63, 182-206 (1988).

22 de Bakker, J. M. et al. Slow conduction in the infarcted human heart. 'Zigzag' course of activation. Circulation 88, 915-926 (1993).

23 Ajijola, O. A. et al. Sympathetic modulation of electrical activation in normal and infarcted myocardium: implications for arrhythmogenesis. Am J Physiol Heart Circ Physiol 312, H608-H621, doi:10.1152/ajpheart.00575.2016 (2017).

$24 \mathrm{Ng}, \mathrm{G}$. A. et al. Sympathetic nerve stimulation produces spatial heterogeneities of action potential restitution. Heart Rhythm 6, 696-706, doi:10.1016/j.hrthm.2009.01.035 (2009).

25 Mantravadi, R. et al. Autonomic nerve stimulation reverses ventricular repolarization sequence in rabbit hearts. Circ Res 100, e72-80, doi:01.RES.0000264101.06417.33 [pii] 10.1161/01.RES.0000264101.06417.33 (2007).

26 Schwartz, P. J., Pagani, M., Lombardi, F., Malliani, A. \& Brown, A. M. A cardiocardiac sympathovagal reflex in the cat. Circ Res 32, 215-220 (1973). 
Kember, G., Armour, J. A. \& Zamir, M. Neural control hierarchy of the heart has not evolved to deal with myocardial ischemia. Physiol Genomics 45, 638-644, doi:10.1152/physiolgenomics.00027.2013 (2013).

28 Zucker, I. H., Patel, K. P. \& Schultz, H. D. Neurohumoral stimulation. Heart failure clinics 8, 87-99, doi:10.1016/j.hfc.2011.08.007 (2012).

29 Wang, H. J., Wang, W., Cornish, K. G., Rozanski, G. J. \& Zucker, I. H. Cardiac sympathetic afferent denervation attenuates cardiac remodeling and improves cardiovascular dysfunction in rats with heart failure. Hypertension 64, 745-755, doi:10.1161/HYPERTENSIONAHA.114.03699 (2014). Allen, E. et al. The electrophysiological effects of nicotinic and electrical stimulation of intrinsic cardiac ganglia in the absence of extrinsic autonomic nerves in the rabbit heart. Heart Rhythm, doi:10.1016/j.hrthm.2018.05.018 (2018).

31 Salavatian, S. et al. Vagal stimulation targets select populations of intrinsic cardiac neurons to control neurally induced atrial fibrillation. Am J Physiol Heart Circ Physiol 311, H1311-H1320, doi:10.1152/ajpheart.00443.2016 (2016).

32 Hamon, D. et al. Premature Ventricular Contraction Coupling Interval Variability Destabilizes Cardiac Neuronal and Electrophysiological Control: Insights From Simultaneous Cardioneural Mapping. Circ Arrhythm Electrophysiol 10, doi:10.1161/CIRCEP.116.004937 (2017).

33 Hoover, D. B. et al. Localization of multiple neurotransmitters in surgically derived specimens of human atrial ganglia. Neuroscience 164, 1170-1179, doi:10.1016/j.neuroscience.2009.09.001 (2009). Takaki, F., Nakamuta, N., Kusakabe, T. \& Yamamoto, Y. Sympathetic and sensory innervation of small intensely fluorescent (SIF) cells in rat superior cervical ganglion. Cell Tissue Res 359, 441-451, doi:10.1007/s00441-014-2051-1 (2015).

35 Nakamura, K. et al. Pathological effects of chronic myocardial infarction on peripheral neurons mediating cardiac neurotransmission. Auton Neurosci 197, 34-40, doi:10.1016/j.autneu.2016.05.001 (2016).

36 Rajendran, P. S. et al. Myocardial infarction induces structural and functional remodelling of the intrinsic cardiac nervous system. J Physiol 594, 321-341, doi:10.1113/JP271165 (2016).

37 Yoshie, K. et al. Cardiac vanilloid receptor-1 afferent depletion enhances stellate ganglion neuronal activity and efferent sympathetic response to cardiac stress. Am J Physiol Heart Circ Physiol 314, H954-H966, doi:10.1152/ajpheart.00593.2017 (2018).

38 Wang, H. J., Rozanski, G. J. \& Zucker, I. H. Cardiac sympathetic afferent reflex control of cardiac function in normal and chronic heart failure states. J Physio/ 595, 2519-2534, doi:10.1113/JP273764 (2017).

39 Herring, N. Autonomic control of the heart: going beyond the classical neurotransmitters. Exp Physiol 100, 354-358, doi:10.1113/expphysiol.2014.080184 (2015).

40 Bardsley, E. N., Davis, H., Buckler, K. J. \& Paterson, D. J. Neurotransmitter Switching Coupled to betaAdrenergic Signaling in Sympathetic Neurons in Prehypertensive States. Hypertension 71, 1226-1238, doi:10.1161/HYPERTENSIONAHA.118.10844 (2018).

41 Weiss, J. N., Chen, P. S., Qu, Z., Karagueuzian, H. S. \& Garfinkel, A. Ventricular fibrillation: how do we stop the waves from breaking? Circ Res 87, 1103-1107 (2000).

42 Garfinkel, A. et al. Preventing ventricular fibrillation by flattening cardiac restitution. Proc Natl Acad Sci U S A 97, 6061-6066, doi:10.1073/pnas.090492697 090492697 [pii] (2000).

43 Kalla, M., Herring, N. \& Paterson, D. J. Cardiac sympatho-vagal balance and ventricular arrhythmia. Auton Neurosci 199, 29-37, doi:10.1016/j.autneu.2016.08.016 (2016).

44 Ishise, H. et al. Time course of sympathovagal imbalance and left ventricular dysfunction in conscious dogs with heart failure. J Appl Physiol 84, 1234-1241 (1998).

45 Motte, S. et al. Respiratory-related heart rate variability in progressive experimental heart failure. Am J Physiol Heart Circ Physiol 289, H1729-1735, doi:10.1152/ajpheart.01129.2004 (2005).

46 Ma, R., Zucker, I. H. \& Wang, W. Central gain of the cardiac sympathetic afferent reflex in dogs with heart failure. Am J Physiol 273, H2664-2671 (1997).

47 Schwartz, P. J., Billman, G. E. \& Stone, H. L. Autonomic mechanisms in ventricular fibrillation induced by myocardial ischemia during exercise in dogs with healed myocardial infarction. An experimental preparation for sudden cardiac death. Circulation 69, 790-800 (1984). 
Cohn, J. N. et al. Plasma norepinephrine as a guide to prognosis in patients with chronic congestive heart failure. N Engl J Med 311, 819-823 (1984).

La Rovere, M. T., Bigger, J. T., Jr., Marcus, F. I., Mortara, A. \& Schwartz, P. J. Baroreflex sensitivity and heart-rate variability in prediction of total cardiac mortality after myocardial infarction. ATRAMI (Autonomic Tone and Reflexes After Myocardial Infarction) Investigators. Lancet 351, 478-484, doi:S0140673697111448 [pii] (1998).

50 Nolan, J. et al. Prospective study of heart rate variability and mortality in chronic heart failure: results of the United Kingdom heart failure evaluation and assessment of risk trial (UK-heart). Circulation 98, 1510-1516 (1998).

51 Somers, V. K., Dyken, M. E., Mark, A. L. \& Abboud, F. M. Sympathetic-nerve activity during sleep in normal subjects. N Engl J Med 328, 303-307, doi:10.1056/NEJM199302043280502 (1993).

52 Verrier, R. L., Muller, J. E. \& Hobson, J. A. Sleep, dreams, and sudden death: the case for sleep as an autonomic stress test for the heart. Cardiovasc Res 31, 181-211 (1996).

53 Schwartz, P. J. et al. Neural control of heart rate is an arrhythmia risk modifier in long QT syndrome. J Am Coll Cardiol 51, 920-929, doi:10.1016/j.jacc.2007.09.069 (2008).

54 Crotti, L. et al. Vagal reflexes following an exercise stress test: a simple clinical tool for gene-specific risk stratification in the long QT syndrome. J Am Coll Cardiol 60, 2515-2524, doi:10.1016/j.jacc.2012.08.1009 (2012).

55 Marban, E., Robinson, S. W. \& Wier, W. G. Mechanisms of arrhythmogenic delayed and early afterdepolarizations in ferret ventricular muscle. J Clin Invest 78, 1185-1192, doi:10.1172/JCl112701 (1986).

56 Shiferaw, Y., Aistrup, G. L. \& Wasserstrom, J. A. Intracellular Ca2+ waves, afterdepolarizations, and triggered arrhythmias. Cardiovasc Res 95, 265-268, doi:10.1093/cvr/cvs155 (2012).

57 Priori, S. G., Mantica, M. \& Schwartz, P. J. Delayed afterdepolarizations elicited in vivo by left stellate ganglion stimulation. Circulation 78, 178-185 (1988).

58 Lubbe, W. F., Podzuweit, T. \& Opie, L. H. Potential arrhythmogenic role of cyclic adenosine monophosphate (AMP) and cytosolic calcium overload: implications for prophylactic effects of betablockers in myocardial infarction and proarrhythmic effects of phosphodiesterase inhibitors. J Am Coll Cardiol 19, 1622-1633, doi:0735-1097(92)90629-2 [pii] (1992).

59 Tsien, R. W. et al. Mechanisms of calcium channel modulation by beta-adrenergic agents and dihydropyridine calcium agonists. J Mol Cell Cardiol 18, 691-710 (1986).

60 Lindemann, J. P., Jones, L. R., Hathaway, D. R., Henry, B. G. \& Watanabe, A. M. beta-Adrenergic stimulation of phospholamban phosphorylation and Ca2+-ATPase activity in guinea pig ventricles. $J$ Biol Chem 258, 464-471 (1983).

61 Hund, T. J. et al. Role of activated CaMKII in abnormal calcium homeostasis and I( $\mathrm{Na})$ remodeling after myocardial infarction: insights from mathematical modeling. J Mol Cell Cardiol 45, 420-428, doi:10.1016/j.yjmcc.2008.06.007 (2008).

62 Bogun, F. et al. Relationship of frequent postinfarction premature ventricular complexes to the reentry circuit of scar-related ventricular tachycardia. Heart Rhythm 5, 367-374, doi:10.1016/j.hrthm.2007.11.026 (2008).

63 Ben-David, J. \& Zipes, D. P. Differential response to right and left ansae subclaviae stimulation of early afterdepolarizations and ventricular tachycardia induced by cesium in dogs. Circulation 78, 1241-1250 (1988).

64 January, C. T. \& Riddle, J. M. Early afterdepolarizations: mechanism of induction and block. A role for L-type Ca2+ current. Circ Res 64, 977-990 (1989).

65 Bers, D. M. \& Morotti, S. Ca(2+) current facilitation is CaMKII-dependent and has arrhythmogenic consequences. Front Pharmacol 5, 144, doi:10.3389/fphar.2014.00144 (2014).

66 Sanguinetti, M. C., Jurkiewicz, N. K., Scott, A. \& Siegl, P. K. Isoproterenol antagonizes prolongation of refractory period by the class III antiarrhythmic agent E-4031 in guinea pig myocytes. Mechanism of action. Circ Res 68, 77-84 (1991).

67 Schwartz, P. J., Verrier, R. L. \& Lown, B. Effect of stellectomy and vagotomy on ventricular refractoriness in dogs. Circ Res 40, 536-540 (1977). 
Bass, B. G. Restitution of the action potential in cat papillary muscle. Am J Physiol 228, 1717-1724, doi:10.1152/ajplegacy.1975.228.6.1717 (1975). Vaseghi, M., Lux, R. L., Mahajan, A. \& Shivkumar, K. Sympathetic stimulation increases dispersion of repolarization in humans with myocardial infarction. Am J Physiol Heart Circ Physiol 302, H18381846, doi:10.1152/ajpheart.01106.2011 (2012). Lang, D. et al. Arrhythmogenic remodeling of beta2 versus beta1 adrenergic signaling in the human failing heart. Circ Arrhythm Electrophysio/ 8, 409-419, doi:10.1161/CIRCEP.114.002065 (2015).

71 Han, J. \& Moe, G. K. Nonuniform Recovery of Excitability in Ventricular Muscle. Circ Res 14, 44-60 (1964).

72 Yagishita, D. et al. Sympathetic nerve stimulation, not circulating norepinephrine, modulates T-peak to T-end interval by increasing global dispersion of repolarization. Circ Arrhythm Electrophysiol 8, 174-185, doi:10.1161/CIRCEP.114.002195 (2015).

73 Nash, M. P. et al. Ventricular activation during sympathetic imbalance and its computational reconstruction. J Appl Physiol (1985) 90, 287-298 (2001).

74 Myles, R. C., Wang, L., Kang, C., Bers, D. M. \& Ripplinger, C. M. Local beta-adrenergic stimulation overcomes source-sink mismatch to generate focal arrhythmia. Circ Res 110, 1454-1464, doi:10.1161/CIRCRESAHA.111.262345 (2012). Stevenson, W. G. Ventricular scars and ventricular tachycardia. Trans Am Clin Climatol Assoc 120, 403-412 (2009). arrhythmogenesis. Cardiovasc Res 62, 323-334, doi:10.1016/j.cardiores.2004.01.033 (2004). Richardson, W. J., Clarke, S. A., Quinn, T. A. \& Holmes, J. W. Physiological Implications of Myocardial Scar Structure. Compr Physiol 5, 1877-1909, doi:10.1002/cphy.c140067 (2015). Luke, R. A. \& Saffitz, J. E. Remodeling of ventricular conduction pathways in healed canine infarct border zones. J Clin Invest 87, 1594-1602, doi:10.1172/JCI115173 (1991). Watkins, H., Ashrafian, H. \& Redwood, C. Inherited cardiomyopathies. N Engl J Med 364, 1643-1656, doi:10.1056/NEJMra0902923 (2011).

80 Janse, M. J., Schwartz, P. J., Wilms-Schopman, F., Peters, R. J. \& Durrer, D. Effects of unilateral stellate ganglion stimulation and ablation on electrophysiologic changes induced by acute myocardial ischemia in dogs. Circulation 72, 585-595 (1985).

81 Li, C. Y. \& Li, Y. G. Cardiac Sympathetic Nerve Sprouting and Susceptibility to Ventricular Arrhythmias after Myocardial Infarction. Cardiol Res Pract 2015, 698368, doi:10.1155/2015/698368 (2015).

82 Parrish, D. C. et al. Transient denervation of viable myocardium after myocardial infarction does not alter arrhythmia susceptibility. Am J Physiol Heart Circ Physiol 314, H415-H423, doi:10.1152/ajpheart.00300.2017 (2018).

83 Gardner, R. T. et al. Targeting protein tyrosine phosphatase sigma after myocardial infarction restores cardiac sympathetic innervation and prevents arrhythmias. Nat Commun 6, 6235, doi:10.1038/ncomms7235 (2015). Ostman, A., Hellberg, C. \& Bohmer, F. D. Protein-tyrosine phosphatases and cancer. Nat Rev Cancer 6, 307-320, doi:10.1038/nrc1837 (2006).

85 Cao, J. M. et al. Nerve sprouting and sudden cardiac death. Circulation research 86, 816-821 (2000).

86 Zhou, S. et al. Mechanisms of cardiac nerve sprouting after myocardial infarction in dogs. Circ Res 95, 76-83, doi:10.1161/01.RES.0000133678.22968.e3 (2004).

87 Hua, F. et al. c-Fos expression in rat brain stem and spinal cord in response to activation of cardiac ischemia-sensitive afferent neurons and electrostimulatory modulation. Am J Physiol Heart Circ Physio/ 287, H2728-2738, doi:10.1152/ajpheart.00180.2004 (2004).

88 Faerman, l. et al. Autonomic neuropathy and painless myocardial infarction in diabetic patients. Histologic evidence of their relationship. Diabetes 26, 1147-1158 (1977).

89 leda, M. et al. Nerve growth factor is critical for cardiac sensory innervation and rescues neuropathy in diabetic hearts. Circulation 114, 2351-2363, doi:10.1161/CIRCULATIONAHA.106.627588 (2006).

90 Jacobson, A. F. et al. Myocardial iodine-123 meta-iodobenzylguanidine imaging and cardiac events in heart failure. Results of the prospective ADMIRE-HF (AdreView Myocardial Imaging for Risk 
Evaluation in Heart Failure) study. J Am Coll Cardiol 55, 2212-2221, doi:10.1016/j.jacc.2010.01.014 (2010).

91 Fallavollita, J. A. et al. Regional myocardial sympathetic denervation predicts the risk of sudden cardiac arrest in ischemic cardiomyopathy. J Am Coll Cardiol 63, 141-149, doi:10.1016/j.jacc.2013.07.096 (2014).

92 Lautamaki, R. et al. Multiparametric molecular imaging provides mechanistic insights into sympathetic innervation impairment in the viable infarct border zone. J Nucl Med 56, 457-463, doi:10.2967/jnumed.114.149971 (2015).

93 Cao, J. M. et al. Relationship between regional cardiac hyperinnervation and ventricular arrhythmia. Circulation 101, 1960-1969 (2000).

94 Karliner, J. S. et al. Beta-adrenergic receptor properties of canine myocardium: effects of chronic myocardial infarction. J Am Coll Cardiol 8, 349-356 (1986).

95 Olivas, A. et al. Myocardial Infarction Causes Transient Cholinergic Transdifferentiation of Cardiac Sympathetic Nerves via gp130. J Neurosci 36, 479-488, doi:10.1523/JNEUROSCI.3556-15.2016 (2016).

96 Kanazawa, H. et al. Heart failure causes cholinergic transdifferentiation of cardiac sympathetic nerves via gp130-signaling cytokines in rodents. J Clin Invest 120, 408-421, doi:10.1172/JCI39778 (2010).

97 Rajendran, P. S. et al. Myocardial infarction induces structural and functional remodelling of the intrinsic cardiac nervous system. The Journal of physiology 594, 321-341, doi:10.1113/JP271165 (2016).

98 Ajijola, O. A. et al. Extracardiac neural remodeling in humans with cardiomyopathy. Circ Arrhythm Electrophysio/ 5, 1010-1116, doi:10.1161/CIRCEP.112.972836 (2012).

99 Ajijola, O. A. et al. Inflammation, oxidative stress, and glial cell activation characterize stellate ganglia from humans with electrical storm. JCI Insight 2, doi:10.1172/jci.insight.94715 (2017).

100 Danson, E. J. \& Paterson, D. J. Reactive oxygen species and autonomic regulation of cardiac excitability. J Cardiovasc Electrophysiol 17 Suppl 1, S104-S112, doi:JCE391 [pii] 10.1111/j.15408167.2006.00391.x (2006).

101 Batulevicius, D., Pauziene, N. \& Pauza, D. H. Architecture and age-related analysis of the neuronal number of the guinea pig intrinsic cardiac nerve plexus. Annals of anatomy = Anatomischer Anzeiger : official organ of the Anatomische Gesellschaft 187, 225-243, doi:10.1016/j.aanat.2005.01.004 (2005).

102 Blomquist, T. M., Priola, D. V. \& Romero, A. M. Source of intrinsic innervation of canine ventricles: a functional study. Am J Physiol 252, H638-644 (1987).

103 Pauza, D. H., Pauziene, N., Pakeltyte, G. \& Stropus, R. Comparative quantitative study of the intrinsic cardiac ganglia and neurons in the rat, guinea pig, dog and human as revealed by histochemical staining for acetylcholinesterase. Annals of anatomy = Anatomischer Anzeiger : official organ of the Anatomische Gesellschaft 184, 125-136, doi:10.1016/S0940-9602(02)80005-X (2002).

104 Coote, J. H. Myths and realities of the cardiac vagus. J Physio/ 591, 4073-4085, doi:10.1113/jphysiol.2013.257758 (2013).

105 Lewis, M. E. et al. Vagus nerve stimulation decreases left ventricular contractility in vivo in the human and pig heart. J Physio/ 534, 547-552 (2001).

106 Singh, S. et al. Topography of cardiac ganglia in the adult human heart. J Thorac Cardiovasc Surg 112, 943-953 (1996).

107 Pardini, B. J., Patel, K. P., Schmid, P. G. \& Lund, D. D. Location, distribution and projections of intracardiac ganglion cells in the rat. J Auton Nerv Syst 20, 91-101 (1987).

108 Berthoud, H. R. \& Neuhuber, W. L. Functional and chemical anatomy of the afferent vagal system. Auton Neurosci 85, 1-17, doi:10.1016/S1566-0702(00)00215-0 (2000).

109 Yamakawa, K. et al. Vagal nerve stimulation activates vagal afferent fibers that reduce cardiac efferent parasympathetic effects. Am J Physiol Heart Circ Physiol 309, H1579-1590, doi:10.1152/ajpheart.00558.2015 (2015).

110 Ardell, J. L. et al. Defining the neural fulcrum for chronic vagus nerve stimulation: implications for integrated cardiac control. J Physiol 595, 6887-6903, doi:10.1113/JP274678 (2017). 
111 Schwartz, P. J. Vagal stimulation for heart failure. Curr Opin Cardiol 26, 51-54, doi:10.1097/HCO.0b013e3283413961 (2011).

112 Schwartz, P. J. Vagal stimulation for heart diseases: from animals to men. - An example of translational cardiology. Circ J 75, 20-27 (2011).

113 Einbrodt. UeberHerzreizungundihrVerhaeltniszum Blutdruck. Akademie der Wissenschaften (Vienna). Sitzungsberichte 38, 345 (1859).

114 Myers, R. W. et al. Beneficial effects of vagal stimulation and bradycardia during experimental acute myocardial ischemia. Circulation 49, 943-947 (1974).

115 Corr, P. B. \& Gillis, R. A. Role of the vagus nerves in the cardiovascular changes induced by coronary occlusion. Circulation 49, 86-97 (1974).

116 Kolman, B. S., Verrier, R. L. \& Lown, B. The effect of vagus nerve stimulation upon vulnerability of the canine ventricle: role of sympathetic-parasympathetic interactions. Circulation 52, 578-585 (1975).

117 Yoon, M. S., Han, J., Tse, W. W. \& Rogers, R. Effects of vagal stimulation, atropine, and propranolol on fibrillation threshold of normal and ischemic ventricles. Am Heart J 93, 60-65 (1977).

118 Kent, K. M., Smith, E. R., Redwood, D. R. \& Epstein, S. E. Electrical stability of acutely ischemic myocardium. Influences of heart rate and vagal stimulation. Circulation 47, 291-298 (1973).

119 Schwartz, P. J. et al. Autonomic mechanisms and sudden death. New insights from analysis of baroreceptor reflexes in conscious dogs with and without a myocardial infarction. Circulation 78, 969-979 (1988).

120 Vanoli, E. et al. Vagal stimulation and prevention of sudden death in conscious dogs with a healed myocardial infarction. Circulation research 68, 1471-1481 (1991).

121 Lakatta, E. G., Maltsev, V. A. \& Vinogradova, T. M. A coupled SYSTEM of intracellular Ca2+ clocks and surface membrane voltage clocks controls the timekeeping mechanism of the heart's pacemaker. Circ Res 106, 659-673, doi:10.1161/CIRCRESAHA.109.206078 (2010).

122 Laurita, K. R. \& Rosenbaum, D. S. Cellular mechanisms of arrhythmogenic cardiac alternans. Prog Biophys Mol Biol 97, 332-347, doi:10.1016/j.pbiomolbio.2008.02.014 (2008).

123 Goldstein, R. E. et al. Influence of atropine and of vagally mediated bradycardia on the occurrence of ventricular arrhythmias following acute coronary occlusion in closed-chest dogs. Circulation 47, 1180-1190 (1973).

124 Kent, K. M., Smith, E. R., Redwood, D. R. \& Epstein, S. E. Beneficial electrophysiologic effects of nitroglycerin during acute myocardial infarction. Am J Cardiol 33, 513-516 (1974).

125 Zuanetti, G., De Ferrari, G. M., Priori, S. G. \& Schwartz, P. J. Protective effect of vagal stimulation on reperfusion arrhythmias in cats. Circulation research 61, 429-435 (1987).

126 Brack, K. E., Coote, J. H. \& Ng, G. A. Vagus nerve stimulation protects against ventricular fibrillation independent of muscarinic receptor activation. Cardiovasc Res 91, 437-446, doi:10.1093/cvr/cvr105 (2011).

127 Kalla, M. et al. Protection against ventricular fibrillation via cholinergic receptor stimulation and the generation of nitric oxide. J Physiol, doi:10.1113/JP271588 (2016).

128 Mesirca, P. et al. The G-protein-gated K+ channel, IKACh, is required for regulation of pacemaker activity and recovery of resting heart rate after sympathetic stimulation. J Gen Physiol 142, 113-126, doi:10.1085/jgp.201310996 (2013).

129 Balligand, J. L., Kelly, R. A., Marsden, P. A., Smith, T. W. \& Michel, T. Control of cardiac muscle cell function by an endogenous nitric oxide signaling system. Proceedings of the National Academy of Sciences of the United States of America 90, 347-351 (1993).

130 Han, X. et al. Muscarinic cholinergic regulation of cardiac myocyte ICa-L is absent in mice with targeted disruption of endothelial nitric oxide synthase. Proc Natl Acad Sci U S A 95, 6510-6515 (1998).

131 Herring, N., Danson, E. J. \& Paterson, D. J. Cholinergic control of heart rate by nitric oxide is site specific. News Physiol Sci 17, 202-206 (2002).

132 Martin, S. R., Emanuel, K., Sears, C. E., Zhang, Y. H. \& Casadei, B. Are myocardial eNOS and nNOS involved in the beta-adrenergic and muscarinic regulation of inotropy? A systematic investigation. Cardiovasc Res 70, 97-106, doi:10.1016/j.cardiores.2006.02.002 (2006). 
$133 \mathrm{Ng}, \mathrm{G}$. A., Brack, K. E. \& Coote, J. H. Effects of direct sympathetic and vagus nerve stimulation on the physiology of the whole heart--a novel model of isolated Langendorff perfused rabbit heart with intact dual autonomic innervation. Exp Physiol 86, 319-329 (2001).

134 Ellenbogen, K. A., Smith, M. L. \& Eckberg, D. L. Increased vagal cardiac nerve traffic prolongs ventricular refractoriness in patients undergoing electrophysiology testing. Am J Cardiol 65, 13451350 (1990).

135 Herring, N., Golding, S. \& Paterson, D. J. Pre-synaptic NO-cGMP pathway modulates vagal control of heart rate in isolated adult guinea pig atria. J Mol Cell Cardiol 32, 1795-1804, doi:10.1006/jmcc.2000.1214 S0022-2828(00)91214-8 [pii] (2000).

136 Herring, N. \& Paterson, D. J. Nitric oxide-cGMP pathway facilitates acetylcholine release and bradycardia during vagal nerve stimulation in the guinea-pig in vitro. J Physio/ 535, 507-518 (2001).

137 Brack, K. E., Patel, V. H., Coote, J. H. \& Ng, G. A. Nitric oxide mediates the vagal protective effect on ventricular fibrillation via effects on action potential duration restitution in the rabbit heart. $J$ Physiol 583, 695-704, doi:jphysiol.2007.138461 [pii] 10.1113/jphysiol.2007.138461 (2007).

138 Mastitskaya, S. et al. Cardioprotection evoked by remote ischaemic preconditioning is critically dependent on the activity of vagal pre-ganglionic neurones. Cardiovasc Res 95, 487-494, doi:10.1093/cvr/cvs212 (2012).

139 Machhada, A. et al. Control of ventricular excitability by neurons of the dorsal motor nucleus of the vagus nerve. Heart Rhythm 12, 2285-2293, doi:10.1016/j.hrthm.2015.06.005 (2015).

140 Shinlapawittayatorn, K. et al. Vagus nerve stimulation initiated late during ischemia, but not reperfusion, exerts cardioprotection via amelioration of cardiac mitochondrial dysfunction. Heart Rhythm 11, 2278-2287, doi:10.1016/j.hrthm.2014.08.001 (2014).

141 De Ferrari, G. M., Vanoli, E., Curcuruto, P., Tommasini, G. \& Schwartz, P. J. Prevention of lifethreatening arrhythmias by pharmacologic stimulation of the muscarinic receptors with oxotremorine. Am Heart J 124, 883-890 (1992).

142 De Ferrari, G. M. et al. Pharmacologic modulation of the autonomic nervous system in the prevention of sudden cardiac death. A study with propranolol, methacholine and oxotremorine in conscious dogs with a healed myocardial infarction. Journal of the American College of Cardiology 22, 283-290 (1993).

143 Farah, C., Michel, L. Y. M. \& Balligand, J. L. Nitric oxide signalling in cardiovascular health and disease. Nat Rev Cardiol 15, 292-316, doi:10.1038/nrcardio.2017.224 (2018).

144 Nicoletti, A. \& Michel, J. B. Cardiac fibrosis and inflammation: interaction with hemodynamic and hormonal factors. Cardiovasc Res 41, 532-543 (1999).

145 Klein, R. M. et al. [Inflammation of the myocardium as an arrhythmia trigger]. Z Kardiol 89 Suppl 3, 24-35 (2000).

146 De Jesus, N. M. et al. Antiarrhythmic effects of interleukin 1 inhibition after myocardial infarction. Heart Rhythm 14, 727-736, doi:10.1016/j.hrthm.2017.01.027 (2017).

147 Calvillo, L. et al. Vagal stimulation, through its nicotinic action, limits infarct size and the inflammatory response to myocardial ischemia and reperfusion. J Cardiovasc Pharmacol 58, 500507, doi:10.1097/FJC.0b013e31822b7204 (2011).

148 Wang, H. et al. Nicotinic acetylcholine receptor alpha7 subunit is an essential regulator of inflammation. Nature 421, 384-388, doi:10.1038/nature01339 (2003).

149 Zhang, Y. et al. Chronic vagus nerve stimulation improves autonomic control and attenuates systemic inflammation and heart failure progression in a canine high-rate pacing model. Circ Heart Fail 2, 692-699, doi:10.1161/CIRCHEARTFAILURE.109.873968 (2009).

150 Ando, M. et al. Efferent vagal nerve stimulation protects heart against ischemia-induced arrhythmias by preserving connexin43 protein. Circulation 112, 164-170,

doi:10.1161/CIRCULATIONAHA.104.525493 (2005).

151 Sabbah, H. N. Electrical vagus nerve stimulation for the treatment of chronic heart failure. Cleve Clin J Med 78 Suppl 1, S24-29, doi:10.3949/ccjm.78.s1.04 (2011).

152 Mohan, R. M. et al. Neuronal nitric oxide synthase gene transfer promotes cardiac vagal gain of function. Circulation research 91, 1089-1091 (2002). 
153 Heaton, D. A. et al. Targeted nNOS gene transfer into the cardiac vagus rapidly increases parasympathetic function in the pig. J Mol Cell Cardiol 39, 159-164, doi:S0022-2828(05)00110-0 [pii] 10.1016/j.yjmcc.2005.03.018 (2005).

154 Billman, G. E., Schwartz, P. J. \& Stone, H. L. The effects of daily exercise on susceptibility to sudden cardiac death. Circulation 69, 1182-1189 (1984).

155 Danson, E. J. \& Paterson, D. J. Enhanced neuronal nitric oxide synthase expression is central to cardiac vagal phenotype in exercise-trained mice. J Physio/ 546, 225-232, doi:PHY_031781 [pii] (2003).

156 Mohan, R. M. et al. Peripheral pre-synaptic pathway reduces the heart rate response to sympathetic activation following exercise training: role of NO. Cardiovasc Res 47, 90-98, doi:S0008-

6363(00)00066-3 [pii] (2000).

157 Behling, A. et al. Cholinergic stimulation with pyridostigmine reduces ventricular arrhythmia and enhances heart rate variability in heart failure. Am Heart J 146, 494-500, doi:10.1016/S00028703(03)00319-3 (2003).

158 Randomised trial of intravenous atenolol among 16027 cases of suspected acute myocardial infarction: ISIS-1. First International Study of Infarct Survival Collaborative Group. Lancet 2, 57-66 (1986).

159 Group, C. T. S. Effects of enalapril on mortality in severe congestive heart failure. Results of the Cooperative North Scandinavian Enalapril Survival Study (CONSENSUS). N Engl J Med 316, 14291435, doi:10.1056/NEJM198706043162301 (1987).

160 Pfeffer, M. A. et al. Effect of captopril on mortality and morbidity in patients with left ventricular dysfunction after myocardial infarction. Results of the survival and ventricular enlargement trial. The SAVE Investigators. N Engl J Med 327, 669-677 (1992).

161 The Cardiac Insufficiency Bisoprolol Study II (CIBIS-II): a randomised trial. Lancet 353, 9-13 (1999).

162 Verma, A. et al. Prevalence, predictors, and mortality significance of the causative arrhythmia in patients with electrical storm. J Cardiovasc Electrophysiol 15, 1265-1270, doi:10.1046/j.15408167.2004.04352.x (2004).

163 Pedersen, C. T. et al. EHRA/HRS/APHRS expert consensus on ventricular arrhythmias. Heart Rhythm 11, e166-196, doi:10.1016/j.hrthm.2014.07.024 (2014).

164 Tung, R. et al. Freedom from recurrent ventricular tachycardia after catheter ablation is associated with improved survival in patients with structural heart disease: An International VT Ablation Center Collaborative Group study. Heart Rhythm 12, 1997-2007, doi:10.1016/j.hrthm.2015.05.036 (2015).

165 Vaseghi, M. et al. Sympathetic innervation of the anterior left ventricular wall by the right and left stellate ganglia. Heart Rhythm 9, 1303-1309, doi:10.1016/j.hrthm.2012.03.052 (2012).

166 Yanowitz, F., Preston, J. B. \& Abildskov, J. A. Functional distribution of right and left stellate innervation to the ventricles. Production of neurogenic electrocardiographic changes by unilateral alteration of sympathetic tone. Circ Res 18, 416-428 (1966).

167 Irie, T. et al. Cardiac sympathetic innervation via middle cervical and stellate ganglia and antiarrhythmic mechanism of bilateral stellectomy. Am J Physiol Heart Circ Physiol 312, H392-H405, doi:10.1152/ajpheart.00644.2016 (2017).

168 Moss, A. J. \& McDonald, J. Unilateral cervicothoracic sympathetic ganglionectomy for the treatment of long QT interval syndrome. N Engl J Med 285, 903-904, doi:10.1056/NEJM197110142851607 (1971).

169 Schwartz, P. J. et al. Left cardiac sympathetic denervation in the therapy of congenital long QT syndrome. A worldwide report. Circulation 84, 503-511 (1991).

170 Wilde, A. A. et al. Left cardiac sympathetic denervation for catecholaminergic polymorphic ventricular tachycardia. N Engl J Med 358, 2024-2029, doi:10.1056/NEJMoa0708006 (2008).

171 Schwartz, P. J. et al. Left cardiac sympathetic denervation in the management of high-risk patients affected by the long-QT syndrome. Circulation 109, 1826-1833, doi:10.1161/01.CIR.0000125523.14403.1E (2004).

172 Vaseghi, M. et al. Cardiac sympathetic denervation in patients with refractory ventricular arrhythmias or electrical storm: intermediate and long-term follow-up. Heart Rhythm 11, 360-366, doi:10.1016/j.hrthm.2013.11.028 (2014). 
173 Vaseghi, M. et al. Cardiac Sympathetic Denervation for Refractory Ventricular Arrhythmias. J Am Coll Cardiol 69, 3070-3080, doi:10.1016/j.jacc.2017.04.035 (2017).

174 Mahajan, A., Moore, J., Cesario, D. A. \& Shivkumar, K. Use of thoracic epidural anesthesia for management of electrical storm: a case report. Heart Rhythm 2, 1359-1362, doi:10.1016/j.hrthm.2005.09.004 (2005).

175 Kamibayashi, T. et al. Thoracic epidural anesthesia attenuates halothane-induced myocardial sensitization to dysrhythmogenic effect of epinephrine in dogs. Anesthesiology 82, 129-134 (1995).

176 Meissner, A. et al. Effects of thoracic epidural anesthesia with and without autonomic nervous system blockade on cardiac monophasic action potentials and effective refractoriness in awake dogs. Anesthesiology 95, 132-138; discussion 136A (2001).

177 Do, D. H. et al. Thoracic Epidural Anesthesia Can Be Effective for the Short-Term Management of Ventricular Tachycardia Storm. J Am Heart Assoc 6, doi:10.1161/JAHA.117.007080 (2017).

178 Meng, L., Tseng, C. H., Shivkumar, K. \& Ajijola, O. Efficacy of Stellate Ganglion Blockade in Managing Electrical Storm: A Systematic Review. JACC Clin Electrophysiol 3, 942-949, doi:10.1016/j.jacep.2017.06.006 (2017).

179 Tzafriri, A. R. et al. Arterial microanatomy determines the success of energy-based renal denervation in controlling hypertension. Sci Transl Med 7, 285ra265, doi:10.1126/scitranslmed.aaa3236 (2015).

180 Huang, B. et al. Renal sympathetic denervation modulates ventricular electrophysiology and has a protective effect on ischaemia-induced ventricular arrhythmia. Exp Physiol 99, 1467-1477, doi:10.1113/expphysiol.2014.082057 (2014).

181 Jiang, W. et al. Comparison between renal denervation and metoprolol on the susceptibility of ventricular arrhythmias in rats with myocardial infarction. Sci Rep 8, 10206, doi:10.1038/s41598018-28562-z (2018).

182 Remo, B. F. et al. Safety and efficacy of renal denervation as a novel treatment of ventricular tachycardia storm in patients with cardiomyopathy. Heart Rhythm 11, 541-546, doi:10.1016/j.hrthm.2013.12.038 (2014).

183 Ukena, C. et al. Renal denervation for treatment of ventricular arrhythmias: data from an International Multicenter Registry. Clin Res Cardiol 105, 873-879, doi:10.1007/s00392-016-1012-y (2016).

184 Armaganijan, L. V. et al. 6-Month Outcomes in Patients With Implantable Cardioverter-Defibrillators Undergoing Renal Sympathetic Denervation for the Treatment of Refractory Ventricular Arrhythmias. JACC Cardiovasc Interv 8, 984-990, doi:10.1016/j.jcin.2015.03.012 (2015).

185 Bhatt, D. L. et al. A controlled trial of renal denervation for resistant hypertension. N Engl J Med 370, 1393-1401, doi:10.1056/NEJMoa1402670 (2014).

186 Townsend, R. R. et al. Catheter-based renal denervation in patients with uncontrolled hypertension in the absence of antihypertensive medications (SPYRAL HTN-OFF MED): a randomised, shamcontrolled, proof-of-concept trial. Lancet 390, 2160-2170, doi:10.1016/S0140-6736(17)32281-X (2017).

187 Liu, S. et al. Ablation of the Ligament of Marshall and Left Stellate Ganglion Similarly Reduces Ventricular Arrhythmias During Acute Myocardial Infarction. Circ Arrhythm Electrophysiol 11, e005945, doi:10.1161/CIRCEP.117.005945 (2018).

188 Yu, L. et al. Optogenetic Modulation of Cardiac Sympathetic Nerve Activity to Prevent Ventricular Arrhythmias. J Am Coll Cardiol 70, 2778-2790, doi:10.1016/j.jacc.2017.09.1107 (2017).

189 Green, A. L. et al. Deep brain stimulation can regulate arterial blood pressure in awake humans. Neuroreport 16, 1741-1745, doi:00001756-200511070-00004 [pii] (2005).

190 Pereira, E. A. et al. Ventral periaqueductal grey stimulation alters heart rate variability in humans with chronic pain. Exp Neurol 223, 574-581, doi:10.1016/j.expneurol.2010.02.004 (2010).

191 Sverrisdottir, Y. B. et al. Differentiated baroreflex modulation of sympathetic nerve activity during deep brain stimulation in humans. Hypertension 63, 1000-1010, doi:10.1161/HYPERTENSIONAHA.113.02970 (2014).

192 Tse, H. F. et al. Thoracic Spinal Cord Stimulation for Heart Failure as a Restorative Treatment (SCS HEART study): first-in-man experience. Heart Rhythm 12, 588-595, doi:10.1016/j.hrthm.2014.12.014 (2015). 
193 Wang, S. et al. Spinal cord stimulation protects against ventricular arrhythmias by suppressing left stellate ganglion neural activity in an acute myocardial infarction canine model. Heart Rhythm 12, 1628-1635, doi:10.1016/j.hrthm.2015.03.023 (2015).

194 Zipes, D. P. et al. Determining the Feasibility of Spinal Cord Neuromodulation for the Treatment of Chronic Systolic Heart Failure: The DEFEAT-HF Study. JACC Heart Fail 4, 129-136, doi:10.1016/j.jchf.2015.10.006 (2016).

195 Vaseghi, M. et al. Parasympathetic dysfunction and antiarrhythmic effect of vagal nerve stimulation following myocardial infarction. JCl Insight 2, doi:10.1172/jci.insight.86715 (2017).

196 Zannad, F. et al. Chronic vagal stimulation for the treatment of low ejection fraction heart failure: results of the NEural Cardiac TherApy foR Heart Failure (NECTAR-HF) randomized controlled trial. Eur Heart J 36, 425-433, doi:10.1093/eurheartj/ehu345 (2015).

197 Gold, M. R. et al. Vagus Nerve Stimulation for the Treatment of Heart Failure: The INOVATE-HF Trial. J Am Coll Cardiol 68, 149-158, doi:10.1016/j.jacc.2016.03.525 (2016).

198 De Ferrari, G. M. et al. Chronic vagus nerve stimulation: a new and promising therapeutic approach for chronic heart failure. Eur Heart J 32, 847-855, doi:10.1093/eurheartj/ehq391 (2011).

199 Premchand, R. K. et al. Autonomic regulation therapy via left or right cervical vagus nerve stimulation in patients with chronic heart failure: results of the ANTHEM-HF trial. J Card Fail 20, 808816, doi:10.1016/j.cardfail.2014.08.009 (2014).

200 Premchand, R. K. et al. Extended Follow-Up of Patients With Heart Failure Receiving Autonomic Regulation Therapy in the ANTHEM-HF Study. J Card Fail 22, 639-642, doi:10.1016/j.cardfail.2015.11.002 (2016).

201 Libbus, I., Nearing, B. D., Amurthur, B., KenKnight, B. H. \& Verrier, R. L. Autonomic regulation therapy suppresses quantitative $T$-wave alternans and improves baroreflex sensitivity in patients with heart failure enrolled in the ANTHEM-HF study. Heart Rhythm 13, 721-728, doi:10.1016/j.hrthm.2015.11.030 (2016).

202 Kember, G., Ardell, J. L., Armour, J. A. \& Zamir, M. Vagal nerve stimulation therapy: what is being stimulated? PLoS One 9, e114498, doi:10.1371/journal.pone.0114498 (2014).

203 Byku, M. \& Mann, D. L. Neuromodulation of the Failing Heart: Lost in Translation? JACC Basic TransI Sci 1, 95-106, doi:10.1016/j.jacbts.2016.03.004 (2016).

204 Nattel, S. \& Dobrev, D. Electrophysiological and molecular mechanisms of paroxysmal atrial fibrillation. Nat Rev Cardiol 13, 575-590, doi:10.1038/nrcardio.2016.118 (2016).

205 Wijffels, M. C., Kirchhof, C. J., Dorland, R. \& Allessie, M. A. Atrial fibrillation begets atrial fibrillation. A study in awake chronically instrumented goats. Circulation 92, 1954-1968 (1995).

206 Haissaguerre, M. et al. Spontaneous initiation of atrial fibrillation by ectopic beats originating in the pulmonary veins. N Engl J Med 339, 659-666, doi:10.1056/NEJM199809033391003 (1998).

207 Pappone, C. et al. Atrial electroanatomic remodeling after circumferential radiofrequency pulmonary vein ablation: efficacy of an anatomic approach in a large cohort of patients with atrial fibrillation. Circulation 104, 2539-2544 (2001).

208 Tan, A. Y., Chen, P. S., Chen, L. S. \& Fishbein, M. C. Autonomic nerves in pulmonary veins. Heart Rhythm 4, S57-60, doi:10.1016/j.hrthm.2006.12.011 (2007).

209 Armour, J. A., Murphy, D. A., Yuan, B. X., Macdonald, S. \& Hopkins, D. A. Gross and microscopic anatomy of the human intrinsic cardiac nervous system. Anat Rec 247, 289-298 (1997).

210 Pauza, D. H., Skripka, V., Pauziene, N. \& Stropus, R. Morphology, distribution, and variability of the epicardiac neural ganglionated subplexuses in the human heart. Anat Rec 259, 353-382 (2000).

211 Allessie, M. A., Lammers, W. J., Bonke, I. M. \& Hollen, J. Intra-atrial reentry as a mechanism for atrial flutter induced by acetylcholine and rapid pacing in the dog. Circulation 70, 123-135 (1984).

212 Coumel, P. et al. [The atrial arrhythmia syndrome of vagal origin]. Arch Mal Coeur Vaiss 71, 645-656 (1978).

213 Andersen, K. et al. Risk of arrhythmias in 52755 long-distance cross-country skiers: a cohort study. Eur Heart J 34, 3624-3631, doi:10.1093/eurheartj/eht188 (2013).

214 Wilhelm, M. Atrial fibrillation in endurance athletes. Eur J Prev Cardiol 21, 1040-1048, doi:10.1177/2047487313476414 (2014). 
215 Abdulla, J. \& Nielsen, J. R. Is the risk of atrial fibrillation higher in athletes than in the general population? A systematic review and meta-analysis. Europace 11, 1156-1159, doi:10.1093/europace/eup197 (2009).

216 Raju, H. \& Kalman, J. M. Management of Atrial Fibrillation in the Athlete. Heart Lung Circ 27, 10861092, doi:10.1016/j.hlc.2018.04.295 (2018).

217 Zipes, D. P., Mihalick, M. J. \& Robbins, G. T. Effects of selective vagal and stellate ganglion stimulation of atrial refractoriness. Cardiovasc Res 8, 647-655 (1974).

218 Liu, L. \& Nattel, S. Differing sympathetic and vagal effects on atrial fibrillation in dogs: role of refractoriness heterogeneity. Am J Physio/ 273, H805-816, doi:10.1152/ajpheart.1997.273.2.H805 (1997).

219 Smeets, J. L., Allessie, M. A., Lammers, W. J., Bonke, F. I. \& Hollen, J. The wavelength of the cardiac impulse and reentrant arrhythmias in isolated rabbit atrium. The role of heart rate, autonomic transmitters, temperature, and potassium. Circ Res 58, 96-108 (1986).

220 Tan, A. Y. et al. Neural mechanisms of paroxysmal atrial fibrillation and paroxysmal atrial tachycardia in ambulatory canines. Circulation 118, 916-925, doi:10.1161/CIRCULATIONAHA.108.776203 (2008).

221 Scherlag, B. J., Yamanashi, W., Patel, U., Lazzara, R. \& Jackman, W. M. Autonomically induced conversion of pulmonary vein focal firing into atrial fibrillation. J Am Coll Cardiol 45, 1878-1886, doi:10.1016/j.jacc.2005.01.057 (2005).

222 Po, S. S. et al. Experimental model for paroxysmal atrial fibrillation arising at the pulmonary veinatrial junctions. Heart Rhythm 3, 201-208, doi:10.1016/j.hrthm.2005.11.008 (2006).

223 Patterson, E. et al. Sodium-calcium exchange initiated by the $\mathrm{Ca} 2+$ transient: an arrhythmia trigger within pulmonary veins. J Am Coll Cardiol 47, 1196-1206, doi:10.1016/j.jacc.2005.12.023 (2006).

224 Patterson, E., Po, S. S., Scherlag, B. J. \& Lazzara, R. Triggered firing in pulmonary veins initiated by in vitro autonomic nerve stimulation. Heart Rhythm 2, 624-631, doi:10.1016/j.hrthm.2005.02.012 (2005).

225 Baez-Escudero, J. L., Keida, T., Dave, A. S., Okishige, K. \& Valderrabano, M. Ethanol infusion in the vein of Marshall leads to parasympathetic denervation of the human left atrium: implications for atrial fibrillation. J Am Coll Cardiol 63, 1892-1901, doi:10.1016/j.jacc.2014.01.032 (2014).

226 Valderrabano, M. et al. Retrograde ethanol infusion in the vein of Marshall: regional left atrial ablation, vagal denervation and feasibility in humans. Circ Arrhythm Electrophysio/ 2, 50-56, doi:10.1161/CIRCEP.108.818427 (2009).

227 Rodriguez-Manero, M., Schurmann, P. \& Valderrabano, M. Ligament and vein of Marshall: A therapeutic opportunity in atrial fibrillation. Heart Rhythm 13, 593-601, doi:10.1016/j.hrthm.2015.10.018 (2016).

228 Jayachandran, J. V. et al. Atrial fibrillation produced by prolonged rapid atrial pacing is associated with heterogeneous changes in atrial sympathetic innervation. Circulation 101, 1185-1191 (2000).

229 Chang, C. M. et al. Nerve sprouting and sympathetic hyperinnervation in a canine model of atrial fibrillation produced by prolonged right atrial pacing. Circulation 103, 22-25 (2001).

230 Nguyen, B. L., Fishbein, M. C., Chen, L. S., Chen, P. S. \& Masroor, S. Histopathological substrate for chronic atrial fibrillation in humans. Heart Rhythm 6, 454-460, doi:10.1016/j.hrthm.2009.01.010 (2009).

231 Stavrakis, S. et al. The role of the autonomic ganglia in atrial fibrillation. JACC Clin Electrophysiol 1, 113, doi:10.1016/j.jacep.2015.01.005 (2015).

232 Stavrakis, S. et al. Low-level transcutaneous electrical vagus nerve stimulation suppresses atrial fibrillation. J Am Coll Cardiol 65, 867-875, doi:10.1016/j.jacc.2014.12.026 (2015).

233 Tsai, C. F. et al. Bezold-Jarisch-like reflex during radiofrequency ablation of the pulmonary vein tissues in patients with paroxysmal focal atrial fibrillation. J Cardiovasc Electrophysiol 10, 27-35 (1999).

234 Hsieh, M. H. et al. Alterations of heart rate variability after radiofrequency catheter ablation of focal atrial fibrillation originating from pulmonary veins. Circulation 100, 2237-2243 (1999).

235 Pappone, C. et al. Pulmonary vein denervation enhances long-term benefit after circumferential ablation for paroxysmal atrial fibrillation. Circulation 109, 327-334, doi:10.1161/01.CIR.0000112641.16340.C7 (2004). 
236 Tan, A. Y. et al. Autonomic innervation and segmental muscular disconnections at the human pulmonary vein-atrial junction: implications for catheter ablation of atrial-pulmonary vein junction. $J$ Am Coll Cardiol 48, 132-143, doi:10.1016/j.jacc.2006.02.054 (2006).

237 Verma, A. et al. Vagal responses induced by endocardial left atrial autonomic ganglion stimulation before and after pulmonary vein antrum isolation for atrial fibrillation. Heart Rhythm 4, 1177-1182, doi:10.1016/j.hrthm.2007.04.023 (2007).

238 Kuck, K. H. et al. Impact of Complete Versus Incomplete Circumferential Lines Around the Pulmonary Veins During Catheter Ablation of Paroxysmal Atrial Fibrillation: Results From the Gap-Atrial Fibrillation-German Atrial Fibrillation Competence Network 1 Trial. Circ Arrhythm Electrophysiol 9, e003337, doi:10.1161/CIRCEP.115.003337 (2016).

239 Katritsis, D. G. et al. Rapid pulmonary vein isolation combined with autonomic ganglia modification: a randomized study. Heart Rhythm 8, 672-678, doi:10.1016/j.hrthm.2010.12.047 (2011).

240 Po, S. S., Nakagawa, H. \& Jackman, W. M. Localization of left atrial ganglionated plexi in patients with atrial fibrillation. J Cardiovasc Electrophysiol 20, 1186-1189, doi:10.1111/j.1540-8167.2009.01515.x (2009).

241 Lemola, K. et al. Pulmonary vein isolation as an end point for left atrial circumferential ablation of atrial fibrillation. J Am Coll Cardiol 46, 1060-1066, doi:10.1016/j.jacc.2005.05.069 (2005).

242 Stabile, G. et al. Is pulmonary vein isolation necessary for curing atrial fibrillation? Circulation 108, 657-660, doi:10.1161/01.CIR.0000086980.42626.34 (2003).

243 Jiang, R. H. et al. Incidence of pulmonary vein conduction recovery in patients without clinical recurrence after ablation of paroxysmal atrial fibrillation: mechanistic implications. Heart Rhythm 11, 969-976, doi:10.1016/j.hrthm.2014.03.015 (2014).

244 Stavrakis, S. \& Po, S. Ganglionated Plexi Ablation: Physiology and Clinical Applications. Arrhythm Electrophysiol Rev 6, 186-190, doi:10.15420/aer2017.26.1 (2017).

245 Pokushalov, E. et al. Selective ganglionated plexi ablation for paroxysmal atrial fibrillation. Heart Rhythm 6, 1257-1264, doi:10.1016/j.hrthm.2009.05.018 (2009).

246 Katritsis, D. G. et al. Autonomic denervation added to pulmonary vein isolation for paroxysmal atrial fibrillation: a randomized clinical trial. J Am Coll Cardiol 62, 2318-2325, doi:10.1016/j.jacc.2013.06.053 (2013).

247 Pokushalov, E. et al. Ganglionated plexus ablation vs linear ablation in patients undergoing pulmonary vein isolation for persistent/long-standing persistent atrial fibrillation: a randomized comparison. Heart Rhythm 10, 1280-1286, doi:10.1016/j.hrthm.2013.04.016 (2013).

248 Pokushalov, E. et al. Ganglionated plexi ablation for longstanding persistent atrial fibrillation. Europace 12, 342-346, doi:10.1093/europace/euq014 (2010).

249 Lin, J. et al. Autonomic mechanism to explain complex fractionated atrial electrograms (CFAE). J Cardiovasc Electrophysiol 18, 1197-1205, doi:10.1111/j.1540-8167.2007.00976.x (2007).

250 Katritsis, D., Giazitzoglou, E., Sougiannis, D., Voridis, E. \& Po, S. S. Complex fractionated atrial electrograms at anatomic sites of ganglionated plexi in atrial fibrillation. Europace 11, 308-315, doi:10.1093/europace/eup036 (2009).

251 Katritsis, D. et al. Autonomic modulation of complex fractionated atrial electrograms in patients with paroxysmal atrial fibrillation. J Interv Card Electrophysiol 31, 217-223, doi:10.1007/s10840-011-95580 (2011).

252 Verma, A. et al. Approaches to catheter ablation for persistent atrial fibrillation. N Engl J Med 372, 1812-1822, doi:10.1056/NEJMoa1408288 (2015).

253 Sha, Y. et al. Low-level right vagal stimulation: anticholinergic and antiadrenergic effects. J Cardiovasc Electrophysiol 22, 1147-1153, doi:10.1111/j.1540-8167.2011.02070.x (2011).

254 Sairaku, A. et al. High-frequency stimulation of the atria increases early recurrence following pulmonary vein isolation in patients with persistent atrial fibrillation. Heart Rhythm 9, 1386-1392, doi:10.1016/j.hrthm.2012.05.014 (2012).

255 Jungen, C. et al. Disruption of cardiac cholinergic neurons enhances susceptibility to ventricular arrhythmias. Nat Commun 8, 14155, doi:10.1038/ncomms14155 (2017). 
256 He, B. et al. Effects of ganglionated plexi ablation on ventricular electrophysiological properties in normal hearts and after acute myocardial ischemia. Int J Cardiol 168, 86-93, doi:10.1016/j.ijcard.2012.09.067 (2013).

257 Hou, Y. et al. Ganglionated plexi modulate extrinsic cardiac autonomic nerve input: effects on sinus rate, conduction, refractoriness, and inducibility of atrial fibrillation. J Am Coll Cardiol 50, 61-68, doi:10.1016/j.jacc.2007.02.066 (2007).

258 Lo, L. W. et al. Paradoxical long-term proarrhythmic effects after ablating the "head station" ganglionated plexi of the vagal innervation to the heart. Heart Rhythm 10, 751-757, doi:10.1016/j.hrthm.2013.01.030 (2013).

259 Matsukawa, T., Sugiyama, Y. \& Mano, T. Age-related changes in baroreflex control of heart rate and sympathetic nerve activity in healthy humans. J Auton Nerv Syst 60, 209-212 (1996).

260 Ben-Menachem, E. Vagus-nerve stimulation for the treatment of epilepsy. Lancet Neurol 1, 477-482 (2002).

261 Li, S. et al. Low-level vagosympathetic stimulation: a paradox and potential new modality for the treatment of focal atrial fibrillation. Circ Arrhythm Electrophysiol 2, 645-651, doi:10.1161/CIRCEP.109.868331 (2009).

262 Yu, L. et al. Low-level vagosympathetic nerve stimulation inhibits atrial fibrillation inducibility: direct evidence by neural recordings from intrinsic cardiac ganglia. J Cardiovasc Electrophysiol 22, 455-463, doi:10.1111/j.1540-8167.2010.01908.x (2011).

263 Stavrakis, S. et al. Low-Level Vagus Nerve Stimulation Suppresses Post-Operative Atrial Fibrillation and Inflammation: A Randomized Study. JACC Clin Electrophysio/ 3, 929-938, doi:10.1016/j.jacep.2017.02.019 (2017).

264 Hardwick, J. C., Ryan, S. E., Beaumont, E., Ardell, J. L. \& Southerland, E. M. Dynamic remodeling of the guinea pig intrinsic cardiac plexus induced by chronic myocardial infarction. Auton Neurosci 181, 4-12, doi:10.1016/j.autneu.2013.10.008 (2014).

265 Fallgatter, A. J. et al. Far field potentials from the brain stem after transcutaneous vagus nerve stimulation. J Neural Transm (Vienna) 110, 1437-1443, doi:10.1007/s00702-003-0087-6 (2003).

$266 \mathrm{Yu}$, L. et al. Low-level transcutaneous electrical stimulation of the auricular branch of the vagus nerve: a noninvasive approach to treat the initial phase of atrial fibrillation. Heart Rhythm 10, 428435, doi:10.1016/j.hrthm.2012.11.019 (2013).

267 Pokushalov, E. et al. Long-Term Suppression of Atrial Fibrillation by Botulinum Toxin Injection Into Epicardial Fat Pads in Patients Undergoing Cardiac Surgery: One-Year Follow-Up of a Randomized Pilot Study. Circ Arrhythm Electrophysiol 8, 1334-1341, doi:10.1161/CIRCEP.115.003199 (2015).

268 de Jong, M. R. et al. Treatment of atrial fibrillation in patients with enhanced sympathetic tone by pulmonary vein isolation or pulmonary vein isolation and renal artery denervation: clinical background and study design : The ASAF trial: ablation of sympathetic atrial fibrillation. Clin Res Cardiol 107, 539-547, doi:10.1007/s00392-018-1214-6 (2018).

269 Vaseghi, M. et al. Supraventricular tachycardia after orthotopic cardiac transplantation. J Am Coll Cardiol 51, 2241-2249, doi:10.1016/j.jacc.2008.02.065 (2008).

270 Bardsley, E. N. et al. RNA Sequencing Reveals Novel Transcripts from Sympathetic Stellate Ganglia During Cardiac Sympathetic Hyperactivity. Sci Rep 8, 8633, doi:10.1038/s41598-018-26651-7 (2018).

271 Davis, H., Bardsley, E. N. \& Paterson, D. J. Transcriptional profiling of stellate ganglia from normotensive and spontaneously hypertensive rat strains. Sci Data 5, 180123, doi:10.1038/sdata.2018.123 (2018).

272 Li, D. \& Paterson, D. J. Cyclic nucleotide regulation of cardiac sympatho-vagal responsiveness. J Physiol 594, 3993-4008, doi:10.1113/JP271827 (2016).

273 Liu, K. et al. Phosphodiesterase $2 \mathrm{~A}$ as a therapeutic target to restore cardiac neurotransmission during sympathetic hyperactivity. JCl Insight 3, doi:10.1172/jci.insight.98694 (2018).

274 Li, D. et al. Efficacy of B-Type Natriuretic Peptide Is Coupled to Phosphodiesterase 2A in Cardiac Sympathetic Neurons. Hypertension 66, 190-198, doi:10.1161/HYPERTENSIONAHA.114.05054 (2015).

275 Bayles, R. G. et al. Transcriptomic and neurochemical analysis of the stellate ganglia in mice highlights sex differences. Sci Rep 8, 8963, doi:10.1038/s41598-018-27306-3 (2018). 
276 Adler-Graschinsky, E. \& Langer, S. Z. Possible role of a beta-adrenoceptor in the regulation of noradrenaline release by nerve stimulation through a positive feed-back mechanism. BrJ Pharmacol 53, 43-50 (1975).

277 Larsen, H. E., Lefkimmiatis, K. \& Paterson, D. J. Sympathetic neurons are a powerful driver of myocyte function in cardiovascular disease. Sci Rep 6, 38898, doi:10.1038/srep38898 (2016).

278 Bardsley, E. N., Larsen, H. E. \& Paterson, D. J. Impaired cAMP-cGMP cross-talk during cardiac sympathetic dysautonomia. Channels (Austin) 11, 178-180, doi:10.1080/19336950.2016.1259040 (2017).

279 Li, D. et al. Abnormal Intracellular Calcium Homeostasis in Sympathetic Neurons From Young Prehypertensive Rats. Hypertension, doi:10.1161/HYPERTENSIONAHA.111.186460 (2012).

280 Shanks, J., Mane, S., Ryan, R. \& Paterson, D. J. Ganglion-specific impairment of the norepinephrine transporter in the hypertensive rat. Hypertension 61, 187-193, doi:10.1161/HYPERTENSIONAHA.112.202184 (2013).

281 Shanks, J. et al. Cardiac sympathetic dysfunction in the prehypertensive spontaneously hypertensive rat. Am J Physiol Heart Circ Physiol 305, H980-986, doi:10.1152/ajpheart.00255.2013 (2013).

282 Shanks, J. \& Herring, N. Peripheral cardiac sympathetic hyperactivity in cardiovascular disease: role of neuropeptides. Am J Physiol Regul Integr Comp Physiol 305, R1411-1420, doi:10.1152/ajpregu.00118.2013 (2013).

283 Paton, J. F., Kasparov, S. \& Paterson, D. J. Nitric oxide and autonomic control of heart rate: a question of specificity. Trends Neurosci 25, 626-631, doi:S0166223602022610 [pii] (2002).

284 Danson, E. J., Li, D., Wang, L., Dawson, T. A. \& Paterson, D. J. Targeting cardiac sympatho-vagal imbalance using gene transfer of nitric oxide synthase. J Mol Cell Cardiol 46, 482-489, doi:S00222828(08)01471-5 [pii] 10.1016/j.yjmcc.2008.12.013 (2009).

285 Larsen, H. E., Bardsley, E. N., Lefkimmiatis, K. \& Paterson, D. J. Dysregulation of Neuronal Ca2+ Channel Linked to Heightened Sympathetic Phenotype in Prohypertensive States. J Neurosci 36, 8562-8573, doi:10.1523/JNEUROSCI.1059-16.2016 (2016).

286 Li, D., Wang, L., Lee, C. W., Dawson, T. A. \& Paterson, D. J. Noradrenergic cell specific gene transfer with neuronal nitric oxide synthase reduces cardiac sympathetic neurotransmission in hypertensive rats. Hypertension 50, 69-74, doi:HYPERTENSIONAHA.107.088591 [pii] 10.1161/HYPERTENSIONAHA.107.088591 (2007).

287 Li, D. et al. Targeted neuronal nitric oxide synthase transgene delivery into stellate neurons reverses impaired intracellular calcium transients in prehypertensive rats. Hypertension 61, 202-207, doi:10.1161/HYPERTENSIONAHA.111.00105 (2013).

288 Lu, C. J. et al. CAPON modulates neuronal calcium handling and cardiac sympathetic neurotransmission during dysautonomia in hypertension. Hypertension 65, 1288-1297, doi:10.1161/HYPERTENSIONAHA.115.05290 (2015).

289 Arking, D. E. et al. A common genetic variant in the NOS1 regulator NOS1AP modulates cardiac repolarization. Nat Genet 38, 644-651, doi:ng1790 [pii] 10.1038/ng1790 (2006).

290 Eijgelsheim, M. et al. Genetic variation in NOS1AP is associated with sudden cardiac death: evidence from the Rotterdam Study. Hum Mol Genet 18, 4213-4218, doi:ddp356 [pii] 10.1093/hmg/ddp356 (2009).

291 Crotti, L. et al. NOS1AP is a genetic modifier of the long-QT syndrome. Circulation 120, 1657-1663, doi:CIRCULATIONAHA.109.879643 [pii] 10.1161/CIRCULATIONAHA.109.879643 (2009).

292 Chang, K. C. et al. CAPON modulates cardiac repolarization via neuronal nitric oxide synthase signaling in the heart. Proc Natl Acad Sci U S A 105, 4477-4482, doi:0709118105 [pii] 10.1073/pnas.0709118105 (2008).

293 Schwartz, P. J. et al. Genotype-phenotype correlation in the long-QT syndrome: gene-specific triggers for life-threatening arrhythmias. Circulation 103, 89-95 (2001).

294 Jaffrey, S. R., Snowman, A. M., Eliasson, M. J., Cohen, N. A. \& Snyder, S. H. CAPON: a protein associated with neuronal nitric oxide synthase that regulates its interactions with PSD95. Neuron 20, 115-124, doi:S0896-6273(00)80439-0 [pii] (1998). 

in cardiac function and pathophysiology. J Physiol 592, 3189-3200, doi:10.1113/jphysiol.2013.270306 (2014).

296 Zhou, S. et al. Spontaneous stellate ganglion nerve activity and ventricular arrhythmia in a canine model of sudden death. Heart Rhythm 5, 131-139, doi:10.1016/j.hrthm.2007.09.007 (2008).

297 Kawashima, T. Anatomy of the cardiac nervous system with clinical and comparative morphological implications. Anat Sci Int 86, 30-49, doi:10.1007/s12565-010-0096-0 (2011).

\section{Acknowledgements}

N.H. is a British Heart Foundation Intermediate Fellow (FS/15/8/3115). D.J.P. acknowledges the NIH SPARC award OT2OD023848 and BHF programme grant RG/17/14/33085. N.H., M.K. and D.J.P. also acknowledge support from the BHF Centre of Research Excellence (RE/08/004), Oxford, UK.

\section{Author contributions}

N.H. and D.J.P. conceived the Review idea. All authors researched the data for the article, provided substantial contributions to discussions of its content, wrote the article and undertook review and/or editing of the manuscript before submission.

\section{Competing interests}

The authors declare no competing interests.

\section{Publisher's note}

Springer Nature remains neutral with regard to jurisdictional claims in published maps and institutional affiliations.

\section{Key points}

- Many primary cardiovascular diseases, such as hypertension, acute myocardial infarction and heart failure, are also diseases of the autonomic nervous system.

- Sympathetic over activity and vagal impairment are a powerful negative prognostic indicator for morbidity and mortality associated with arrhythmia and sudden cardiac death.

- Emerging evidence suggests neuromodulation therapy might provide important clinical utility in the management and prevention of lethal arrhythmia.

- Neuromodulation device therapy has yielded conflicting and disappointing results in clinical trials, which might be related to stimulation parameters and/or lack of site-specific targeting and appreciation of the complex neural circuitry driving postsynaptic excitability.

- Surgical resection or ablation of specific ganglion, in particular the stellate ganglion, has produced encouraging therapeutic benefits in patients with sympathetic hyperactivity who are prone to arrhythmia. 
- Understanding the relationship between neural circuitry and the molecular pathways underpinning abnormal neurotransmission to cardiac electrophysiology is essential to improve neuromodulation therapy.

Figure 1 | Neuromodulation targets for treating cardiac arrhythmia. Schematic representation of the extrinsic and intrinsic autonomic nervous system and the different targets for autonomic nervous system modulation in the treatment of cardiac arrhythmias. AF, atrial fibrillation; MI, myocardial infarction; PVI, pulmonary vein isolation; VF, ventricular fibrillation; VT, ventricular tachycardia. Modified from Krul et al ${ }^{4}$.

Figure 2 | The cardio-neural hierarchy a | Schema of the cardiac nervous system. b | Simplified construct of neurohumoral control and functional organization of cardiac innervation. $\beta_{1}, \beta_{1}$-adrenergic receptor; $C$, cervical; DRG, dorsal root ganglion; $\mathrm{G}_{\mathrm{i}}$, inhibitory $\mathrm{G}$ protein; $\mathrm{G}_{s}$, stimulatory $\mathrm{G}$ protein; ICNS, intrinsic cardiac nervous system; L, lumbar; M2, muscarinic acetylcholine receptor M2; T, thoracic. Modified from Shivkumar et al ${ }^{9}$.

Figure 3 | Cardiac autonomic innervation in humans. Diagrams showing the complexity of the human autonomic cardiac nervous system, with great arterial bifurcations viewed from the ventral (part a) and dorsal (part b) aspects. The sympathetic, parasympathetic vagal, and mixed nerves are coloured orange, green, and purple, respectively. Black stars, squares, and circles indicate the inlet/outlet of the cardiac nerve/branches from the arterial part of the cardiac hilum itself, and venous parts of the cardiac hilum. From

\section{Kawashima ${ }^{297}$. Redrawn with permission of Elsevier.}

Figure 4 | Cardiac autonomic innervation a |Posterior view of the human heart and major vessels illustrating the locations of posterior atrial and ventricular ganglionated plexi (GP). Note the mediastinal nerves coursing adjacent to the aortic root and joining the two superior atrial ganglionated plexuses. Positions of the superior vena cava, inferior vena cava, right ventricle (RV), left atrium (LA) and left ventricle (LV) are shown. $\mathbf{b}$ | Confocal image of intrinsic neurons in mouse heart labelled with multicolour adenoassociated viral technique. c | Embryonic day 18.5 mouse heart stained for myocardium (red) and tyrosine hydroxylase (green) optically cleared using 3-Disco, showing that before birth, the sympathetic innervation has already formed a dense network on the epicardial surface of the ventricle and is starting to penetrate the myocardial wall. Panel $a$ is from Armour et $\mathrm{al}^{209}$. Panel $\mathrm{b}$ is from Rajendran et $\mathrm{al}^{36}$ with permission. Panel c is Courtesy of Dr Juanjuan Zhao and Dr Mathilda Mommersteeg, Department of Physiology, Anatomy \& Genetics, University of Oxford.

Figure 5 | The autonomic nervous system in cardiac a | Cardiac disease progression. Distribution of autonomic nerves in the heart and disease progression from healthy state to heart failure, in which neural remodelling occurs. As disease progresses from healthy state to pre-diseased state, abnormal increases in intracellular $\mathrm{Ca}^{2+}$ linked to increased neurotransmission can be detected in sympathetic neurones ${ }^{279,285}$. This 
cellular phenotype occurs before the overt clinical signs of hypertension and heart failure, and probably contributes to the sympathetic hyper-responsiveness when the disease is clinically evident. In parallel, parasympathetic impairment is also an early hallmark of autonomic impairment that further facilitates sympathetic dysfunction ${ }^{111-112,284}$. Together, dysautonomia associated with both branches of the autonomic nervous system provide a trigger for abnormal conduction and rhythm disturbance ${ }^{112,120}$. Modified from Li \& Paterson ${ }^{272}$ and Zhang et $a^{295} \mathbf{b}$ | Schematic showing the site of left cardiac sympathetic denervation. $\mathbf{c}$ | Implantable cardioverter defibrillator (ICD) shock free survival is better after bilateral cardiac sympathetic denervation (CSD) than after left CSD in patients with refractory ventricular tachycardia (VT) and structural heart disease. From Vaseghi et al $^{173}$.

Figure 6 | Transcriptomics for the discovery of novel neuronal targets Example of a study using RNA sequencing of stellate ganglia from normal rats and from rats with sympathetic hyperactivity, with validation in human samples and proposed disease model and therapeutic targets a | Gene ontology (GO) analysis of the differentially expressed genes showed that the top two over-represented 'molecular function' GO groups included 'extracellular ligand-gated ion channel activity' and 'phosphoric ester hydrolase activity'. Venn diagrams show upregulated (green) and downregulated genes (blue) in each GO group. Heat-map analysis of the RNA sequencing data showing the expression levels of differentially expressed genes, where green and blue represent upregulated and downregulated genes, respectively. $\mathbf{b}$ | The differential regulation of genes in the GO group of 'extracellular ligand-gated ion channel activity' was then validated with quantitative reverse transcription PCR in stellate ganglia from normal rats and rats with sympathetic hyperactivity, and in human samples of stellate ganglia. c | Kyoto Encyclopedia of Genes and Genome (KEGG) enrichment pathway analysis was used to identify relevant intracellular signalling pathways associated with the observed transcriptomic changes in rat stellate ganglia, revealing three KEGG pathways that were relevant to post-synaptic postganglionic signalling: 'circadian entrainment', 'dopaminergic synapse' and 'glutamatergic synapse'. The Venn diagram (left panel) represents the upregulated (green) and downregulated (blue) genes and the overlap between each of the KEGG groups. Finally, the KEGG signalling pathways were integrated in a cell model (right panel) highlighting the possible involvement of the identified transcriptomic changes on signalling and the link to abnormal regulation of intracellular $\mathrm{Ca}^{2+}$ levels and neurotransmission. For full methods see the original publication by Bradsley et al. ${ }^{270}$. Modified from Bardsley et al ${ }^{270}$ with permission of authors.

Figure 7 | Neurotransmitter switching in sympathetic neurons in prehypertension. a | In healthy postganglionic sympathetic neurons , $\mathrm{Ca}^{2+}$-dependent exocytosis facilitates the release of noradrenaline (NA), which activates $\beta_{1}$-adrenergic receptors $\left(\beta_{1} A R\right)$ and $\beta_{2} A R$ in cardiomyocytes. The increase in extracellular NA also activates presynaptic $\alpha_{2}$-adrenergic receptors $\left(\alpha_{2} A R\right)$, which leads to a reduction in adenylyl cyclase activity in postganglionic sympathetic neurons through activation of inhibitory Ga $\alpha_{i}$ proteins. 
Acute regulation of cAMP levels is mediated by cyclic nucleotide phosphodiesterases (PDEs). cAMPdependent protein kinase $\mathrm{A}$ (PKA) activity increases the levels of intracellular $\mathrm{Ca}^{2+}$ through phosphorylation of the $\mathrm{N}$-type $\mathrm{Ca}^{2+}$ channel $\left(/_{\mathrm{caN}}\right)$ and the regulation of endoplasmic reticulum $\mathrm{Ca}^{2+}$ stores and mitochondrial $\mathrm{Ca}^{2+}$ release. $\mathbf{b}$ | In prehypertensive conditions, $\mathrm{Ca}^{2+}$-dependent exocytosis in postganglionic sympathetic neurons facilitates the release of NA, neuropeptide Y (NPY) and adrenaline (Adr), which activate presynaptic $\beta_{1} A R$ and $\beta_{2} A R$ leading to higher cAMP generation, PKA activity and intracellular $\mathrm{Ca}^{2+}$ levels than in healthy neurons, thereby facilitating neurotransmission to the cardiomyocytes in a potentiating manner. This positive feedback occurs preferentially via $\beta_{2} A R$ activation. Catecholamines can also come from the blood. Modified from Bardsley et $\mathrm{al}^{40}$ with permission.

Figure 9 | Potential mechanisms of cardiac sympatho-vagal transmission regulation. a |Schematic representation of potential signal transduction pathways mediated by natriuretic peptides and nitric oxide (NO)-CAPON (also known as NOS1AP) in depolarized cardiac stellate neurons that target cGMP. Increased levels of CAPON in stellate neurons decreases neurotransmission and postsynaptic excitability by causing cGMP-dependent modulation of intracellular free $\mathrm{Ca}^{2+}$ concentration in stellate neurons. In cardiomyocytes, increased levels of CAPON decrease activation of the L-type $\mathrm{Ca}^{2+}$ current $\left(/_{\mathrm{CaL}}\right)$ and increased delayed rectifier $\mathrm{K}^{+}$current $\left(/_{\mathrm{Kr}}\right)$ via a NO-dependent pathway that shortens the action potential duration. Targeting presynaptic and postsynaptic sites with CAPON gene therapy might have therapeutic utility in patients with certain mutations associated with long QT syndrome. $\mathbf{b}$ | Representative action potential recordings in a CAPON-overexpressing cardiomyocyte showing that administration of the NO synthase inhibitor L-arginine methyl ester (L-NAME) reversal of action potential duration abbreviation. Modified from Li \& Paterson ${ }^{272}$ and Chang et al $^{292}$.

\section{Glossary terms}

Stellate ganglia

Refractory period

Electrical alternans

Cholinergic neurons

Optogenetic

VT storm

Evoked potentials

CLARITY 